\title{
A FINITE ELEMENT SPLITTING EXTRAPOLATION FOR SECOND ORDER HYPERBOLIC EQUATIONS*
}

\author{
XIAOMING $\mathrm{HE}^{\dagger}$ AND TAO LÜ‡
}

\begin{abstract}
Splitting extrapolation is an efficient technique for solving large scale scientific and engineering problems in parallel. This article discusses a finite element splitting extrapolation for second order hyperbolic equations with time-dependent coefficients. This method possesses a higher degree of parallelism, less computational complexity, and more flexibility than Richardson extrapolation while achieving the same accuracy. By means of domain decomposition and isoparametric mapping, some grid parameters are chosen according to the problem. The multiparameter asymptotic expansion of the $d$-quadratic finite element error is also established. The splitting extrapolation formulas are developed from this expansion. An approximation with higher accuracy on a globally fine grid can be computed by solving a set of smaller discrete subproblems on different coarser grids in parallel. Some a posteriori error estimates are also provided. Numerical examples show that this method is efficient for solving discontinuous problems and nonlinear hyperbolic equations.
\end{abstract}

Key words. extrapolation, asymptotic expansion, finite elements, parallel algorithm, a posteriori error estimate

AMS subject classifications. 65B05, 65M60, 65M12, 35R05

DOI. $10.1137 / 070703090$

1. Introduction. The extrapolation techniques for approximations with a higher order of accuracy can be dated back to the 17th century [23]. One of the most important extrapolation techniques is Richardson extrapolation, which is an efficient acceleration method to improve the accuracy of an approximation. Therefore, a lot of work has been done on this method. Richardson extrapolation was used to accelerate the convergence of finite difference methods $[15,41,44]$ and finite element methods $[6,14,20,29,31,50,53]$. In addition, it was applied to many numerical methods for integrations [13], integral equations [17, 32, 34, 35], flow problems [4, 21], statistical inference [5], and many other problems. There has also been a lot of work contributed to the development, generalization, and analysis of different kinds of extrapolation methods; see [7, 9, 24, 25, 42, 45, 48, 49, 51] and the references therein.

In 1983, based on Richardson extrapolation, the idea of splitting extrapolation was developed by Lin and Lü [30] and applied to multiple numerical integrations, multidimensional integral equations, and the difference method for solving Poisson's equation. For each case, they also gave the corresponding error estimates. Since then Lin and Zhu [33], Neittaanmäki and Lin [43], Lü, Shih, and Liem [39], Rüde and Zhou [47], Huang and Lü [19], and others have applied splitting extrapolation to different kinds of algorithms, such as high-dimensional numerical quadrature, finite difference methods and numerical methods for integral equations. Lü, Shih, and Liem (see $[28,40]$ ) published two monographs on splitting extrapolation, and Rüde [46] reviewed the monograph [28] in SIAM Review in 1997.

*Received by the editors September 17, 2007; accepted for publication (in revised form) September 3, 2009; published electronically November 25, 2009. This work was partially supported by NSF grant DMS-0713763 and the National Science Foundation of China (10671136).

http://www.siam.org/journals/sisc/31-6/70309.html

${ }^{\dagger}$ Corresponding author. Department of Mathematics, Virginia Tech, Blacksburg, VA 24061 (xiaoming@vt.edu).

${ }^{\ddagger}$ College of Mathematics, Sichuan University, Chengdu, Sichuan 610064, People’s Republic of China (lutao@public.cd.sc.cn). 
Now we describe the basic idea of splitting extrapolation based on Richardson extrapolation. We first choose some independent grid parameters, say $h_{1}, \ldots, h_{k}$, for a method of approximation. Suppose the approximation is $u\left(h_{1}, \ldots, h_{k}\right)$ and we can establish a multiparameter asymptotic expansion of the error with respect to the independent parameters. Then we can construct a linear combination of the approximations with different values of the independent parameters, such as $u\left(h_{1}, \ldots, h_{k}\right)$, $u\left(\frac{h_{1}}{2}, \ldots, h_{k}\right), \ldots, u\left(h_{1}, \ldots, \frac{h_{k}}{2}\right)$, to cancel out the lower order terms in the multiparameter asymptotic expansion. Then we get a new approximation, which has the same order of accuracy as the higher order remainder in the multiparameter asymptotic expansion. It is therefore more accurate than any of the approximations in the linear combination. This process can be repeated as long as we can show the existence of higher order terms in the expansion.

Splitting extrapolation has several advantages. First, the algorithm possesses a high degree of parallelism and a high order of accuracy. Also, its computational complexity is less than that of Richardson extrapolation [46]. Second, the design of the independent parameters gives us flexibility in choosing different kinds of meshes, especially when it is combined with domain decomposition. Last but not least, the splitting extrapolation formulas can produce an approximation on the global fine grid instead of the coarse grid on which we apply the original method of approximation; see section 5 in this paper or $[18,28,37,38,40]$. These advantages become more apparent as the size of the problem increases and more independent grid parameters are chosen with domain decomposition.

In summary, splitting extrapolation is an efficient parallel algorithm that overcomes the curse of dimensionality to some extent. The key steps of splitting extrapolation are to establish the multiparameter asymptotic expansion of the error and develop the splitting extrapolation formulas. We refer the readers to $[8,18,19,27$, $28,30,33,36,37,38,39,40,43,46,47,54]$ for more background material about splitting extrapolation.

Finite element splitting extrapolation based on domain decomposition is one of the important applications of splitting extrapolation. By using the algorithm, the independent grid parameters are designed with an initial domain decomposition according to the dimension and interface of the problem, the shape and size of the domain, and the computers used. Therefore, the algorithm combines the advantages of domain decomposition and splitting extrapolation and can be applied to interface problems with discontinuous coefficients. Since Lü [36] proposed the finite element splitting extrapolation in 1987, it has been successfully applied to different types of problems [8, 18, 27, 37, 38, 54].

A lot of work has been contributed to the Galerkin method for solving second order hyperbolic equations $[1,2,3,11,12,16,52]$, but there are few papers about the extrapolation of this method. In this paper we will investigate the finite element splitting extrapolation for solving second order hyperbolic equations based on domain decomposition and $d$-quadratic isoparametric finite elements.

This paper is organized as follows: in section 2, we present the model problem and its discrete schemes; in section 3, we establish the multiparameter asymptotic expansion of the semidiscrete $d$-quadratic isoparametric finite element error; in section 4 , we establish the multiparameter asymptotic expansion of the fully discrete $d$-quadratic isoparametric finite element error; in section 5 , we develop the corresponding splitting extrapolation formulas; in section 6 , we present some a posteriori error estimates; and finally in section 7 , we present four numerical examples. 
2. Second order hyperbolic equation and its discrete schemes. In this section we will present the weak formulation, the semidiscrete approximation, and the fully discrete approximation $[10,12,22,26]$ for the following second order hyperbolic equation:

$$
\left\{\begin{array}{lll}
u_{t t}(t, x)-\sum_{i, j=1}^{d} D_{i}\left(a_{i j}(t, x) D_{j} u(t, x)\right)+q(t, x) u(t, x)=f(t, x) & \text { on } & Q_{T}=[0, T] \times \Omega, \\
u(t, x)=0 & \text { on } & \Sigma_{T}=[0, T] \times \partial \Omega \\
u(0, x)=u_{0}(x), u_{t}(0, x)=u_{1}(x) & \text { on } & \bar{\Omega} .
\end{array}\right.
$$

Here $x=\left(x_{1}, \cdots, x_{d}\right), D_{i}=\frac{\partial}{\partial x_{i}}$, and the domain $\Omega \subset \mathbb{R}^{d}(d=2,3)$ is an open set with piecewise smooth boundary.

Remark 2.1. Without loss of generalization, we consider only the hyperbolic equations with homogeneous Dirichlet boundary condition here. The splitting extrapolation can also be used to handle the problems with nonhomogeneous Dirichlet boundary condition and mixed boundary condition by either modifying the weak formulation or reducing the problems to homogeneous problems via the usual homogenization technique based on a change of variable.

First, we construct a nonoverlapping initial domain decomposition $\bar{\Omega}=\bigcup_{k=1}^{m} \bar{\Omega}_{k}$, where $\Omega_{k}(k=1, \ldots, m)$ satisfy the usual compatibility conditions. By the standard $d$-quadratic isoparametric mapping $[10,18,38]$, there are translated unit cubes $\hat{\Omega}_{k}(k=1, \ldots, m) \subset \mathbb{R}^{d}$ and one-to-one $d$-quadratic isoparametric mappings $\Psi_{k}$ : $\Omega_{k} \rightarrow \hat{\Omega}_{k}$ such that $\left\{\Psi_{k}^{-1}\right\}$ are sufficiently smooth. Let $\hat{\Omega}$ denote the open set satisfying $\overline{\hat{\Omega}}=\bigcup_{k=1}^{m} \overline{\hat{\Omega}}_{k}$. Let $\hat{\Im}_{k}^{h}(k=1, \ldots, m)$ be a uniform cuboid partition with grid parameter $\hat{h}_{k j}(j=1, \ldots, d)$ on $\hat{\Omega}_{k}$ such that $\hat{\Im}^{h}=\bigcup_{k=1}^{m} \hat{\Im}_{k}^{h}$ is a piecewise uniform cuboid partition on $\hat{\Omega}$. In order to avoid hanging nodes on the boundaries of $\hat{\Omega}_{k}(k=1, \ldots, m)$, some $\hat{h}_{k j}$ need to be the same so that the partitions $\hat{\Im}_{k}^{h}$ are geometrically conforming. We can combine those $\hat{h}_{k j}$ into one grid parameter. Hence there are only $l(l<m d)$ independent grid parameters whose values can be chosen independently such that there are no hanging nodes on the boundaries of $\hat{\Omega}_{k}(k=1, \ldots, m)$. Example 1 in section 7 provides an example of designing independent grid parameters. Also, we choose a time step size $\tau$, so there are $l+1$ independent grid parameters which are denoted by $\hat{h}_{1}, \ldots, \hat{h}_{l+1}$. Here we use $\hat{h}_{l+1}$ to denote $\tau$. By the $d$-quadratic isoparametric mapping, (2.1) is converted into the following problem:

$$
\begin{cases}\hat{u}_{t t}(t, \hat{x})-\sum_{i, j=1}^{d} \hat{D}_{i}\left(\hat{a}_{i j}(t, \hat{x}) \hat{D}_{j} \hat{u}(t, \hat{x})\right)+\hat{q}(t, \hat{x}) \hat{u}(t, \hat{x})=\hat{f}(t, \hat{x}) & \text { on } \quad \hat{Q}_{T}=[0, T] \times \hat{\Omega} \\ \hat{u}(t, \hat{x})=0 & \text { on } \quad \hat{\Sigma}_{T}=[0, T] \times \partial \hat{\Omega} \\ \hat{u}(0, \hat{x})=\hat{u}_{0}(\hat{x}), \hat{u}_{t}(0, \hat{x})=\hat{u}_{1}(\hat{x}) & \text { on } \quad \hat{\hat{\Omega}}\end{cases}
$$

Here $\hat{x}=\left(\hat{x}_{1}, \ldots, \hat{x}_{d}\right), \hat{D}_{i}=\frac{\partial}{\partial \hat{x}_{i}}$, and $\left.\hat{u}\right|_{\hat{\Omega}_{k}}=\Psi_{k}\left(\left.u\right|_{\Omega_{k}}\right)$. 
We define

$$
\begin{aligned}
& A(t ; \hat{u}, \hat{v})=\int_{\hat{\Omega}}\left(\sum_{i, j=1}^{d} \hat{a}_{i j} \hat{D}_{i} \hat{u} \hat{D}_{j} \hat{v}+\hat{q} \hat{u} \hat{v}\right) \mathrm{d} \hat{x} \quad \forall \hat{u}, \hat{v} \in H^{1}(\hat{\Omega}), \\
& A^{\prime}(t ; \hat{u}, \hat{v})=\int_{\hat{\Omega}}\left(\sum_{i, j=1}^{d} \frac{\partial \hat{a}_{i j}}{\partial t} \hat{D}_{i} \hat{u} \hat{D}_{j} \hat{v}+\frac{\partial \hat{q}}{\partial t} \hat{u} \hat{v}\right) \mathrm{d} \hat{x} \quad \forall \hat{u}, \hat{v} \in H^{1}(\hat{\Omega})
\end{aligned}
$$

and assume the coercivity and boundedness as follows:

$$
\begin{aligned}
& A(t ; \hat{u}, \hat{u}) \geq \mu_{1}\|\hat{u}\|_{1, \hat{\Omega}}^{2} \quad \forall \hat{u} \in H^{1}(\hat{\Omega}), \forall t \in[0, T], \\
& |A(t ; \hat{u}, \hat{v})| \leq \mu_{2}\|\hat{u}\|_{1, \hat{\Omega}}\|\hat{v}\|_{1, \hat{\Omega}} \quad \forall \hat{u}, \hat{v} \in H^{1}(\hat{\Omega}), \forall t \in[0, T], \\
& \left|A^{\prime}(t ; \hat{u}, \hat{v})\right| \leq \mu_{3}\|\hat{u}\|_{1, \hat{\Omega}}\|\hat{v}\|_{1, \hat{\Omega}} \quad \forall \hat{u}, \hat{v} \in H^{1}(\hat{\Omega}), \forall t \in[0, T] .
\end{aligned}
$$

For a given Banach space $B$, we define

$$
\begin{aligned}
L^{p}(0, T ; B)= & \left\{\hat{u}(t, \cdot):[0, T] \rightarrow B \text { s.t. }\|\hat{u}\|=\left(\int_{0}^{T}\|\hat{u}(t, \cdot)\|_{B}^{p} d t\right)^{\frac{1}{p}}<\infty\right\}, \\
H^{m}(0, T ; B)= & \left\{\hat{u}(t, \cdot):[0, T] \rightarrow B \text { s.t. } \frac{\partial^{i} \hat{u}}{\partial t^{i}} \in L^{2}(0, T ; B), i=0, \ldots, m\right\}, \\
C^{k}(0, T ; B)= & \{\hat{u}(t, \cdot):[0, T] \rightarrow B \text { s.t. } \hat{u}(t) \text { has up to } k \text { th order continuous } \\
& \text { derivative for t }\} .
\end{aligned}
$$

We use $\hat{S}_{0}^{h} \subset H_{0}^{1}(\hat{\Omega}) \cap C(\hat{\Omega})$ to denote the $d$-quadratic finite element space under the partition $\hat{\Im}^{h}$.

It is well known that the weak formulation of our problem can be obtained as follows: Find $\hat{u} \in H^{2}\left(0, T ; H_{0}^{1}(\hat{\Omega})\right)$ satisfying

$$
\left\{\begin{array}{l}
\left(\hat{u}_{t t}, \hat{v}\right)+A(t ; \hat{u}, \hat{v})=(\hat{f}, \hat{v}) \\
\hat{u}(0, \hat{x})=\hat{u}_{0}(\hat{x}), \hat{u}_{t}(0, \hat{x})=\hat{u}_{1}(\hat{x})
\end{array} \quad \forall \hat{v} \in H_{0}^{1}(\hat{\Omega})\right.
$$

Next the semidiscrete approximation can be obtained as follows [12, 26]: Find $\overline{\hat{u}} \in$ $H^{2}\left(0, T ; \hat{S}_{0}^{h}\right)$ satisfying

$$
\begin{cases}\left(\overline{\hat{u}}_{t t}, \hat{v}\right)+A(t ; \overline{\hat{u}}, \hat{v})=(\hat{f}, \hat{v}) & \forall \hat{v} \in \hat{S}_{0}^{h} \\ (\overline{\hat{u}}(0, \hat{x}), \hat{v})=\left(\hat{u}_{0}(\hat{x}), \hat{v}\right),\left(\overline{\hat{u}}_{t}(0, \hat{x}), \hat{v}\right)=\left(\hat{u}_{1}(\hat{x}), \hat{v}\right) & \forall \hat{v} \in \hat{S}_{0}^{h}\end{cases}
$$

Let $\hat{P}_{h}$ denote the orthogonal projection operator mapping $L^{2}(\hat{\Omega})$ to $\hat{S}_{0}^{h}$. Then from (2.7), we get

$$
\overline{\hat{u}}(0, \hat{x})=\hat{P}_{h} \hat{u}_{0}(\hat{x}), \overline{\hat{u}}_{t}(0, \hat{x})=\hat{P}_{h} \hat{u}_{1}(\hat{x}) .
$$

Let $\hat{h}_{l+1}=\tau=\frac{T}{N}$ denote the time step size, $t_{n}=n \tau$, and $w^{n}=w(n \tau, \hat{x})$. Then a well-known fully discrete approximation $[12,26]$ can be obtained as follows: Find $\hat{U}^{n} \in \hat{S}_{0}^{h}, n=1, \ldots, N$, satisfying

$$
\left\{\begin{array}{l}
\left(\bar{\partial}_{t t} \hat{U}^{n}, \hat{v}\right)+A\left(t_{n} ; \hat{U}^{n, \frac{1}{4}}, \hat{v}\right)=\left(\hat{f}^{n}, \hat{v}\right) \quad \forall \hat{v} \in \hat{S}_{0}^{h} \\
\left(\hat{U}^{0}, \hat{v}\right)=\left(\hat{u}_{0}, \hat{v}\right) \quad \forall \hat{v} \in \hat{S}_{0}^{h}, \\
\left(\hat{U}^{1}, \hat{v}\right)=\left(\hat{u}_{0}, \hat{v}\right)+\tau\left(\hat{u}_{1}, \hat{v}\right)+\frac{\tau^{2}}{2}\left[\left(\hat{f}^{0}, \hat{v}\right)-A\left(0 ; \hat{P}_{h} \hat{u}_{0}, \hat{v}\right)\right] \\
+\frac{\tau^{3}}{6}\left[\left(\hat{f}_{t}^{0}, \hat{v}\right)-A^{\prime}\left(0 ; \hat{P}_{h} \hat{u}_{0}, \hat{v}\right)-A\left(0 ; \hat{P}_{h} \hat{u}_{1}, \hat{v}\right)\right] \quad \forall \hat{v} \in \hat{S}_{0}^{h}
\end{array}\right.
$$

Copyright $@$ by SIAM. Unauthorized reproduction of this article is prohibited. 
Here $\bar{\partial}_{t t} \hat{U}^{n}=\frac{\hat{U}^{n+1}-2 \hat{U}^{n}+\hat{U}^{n-1}}{\tau^{2}}, \hat{U}^{n, \frac{1}{4}}=\frac{\hat{U}^{n+1}+2 \hat{U}^{n}+\hat{U}^{n-1}}{4}$.

It is well known that the standard Galerkin method with only one grid parameter can be used to get an approximation and Richardson extrapolation can be applied to this approximation. Our goal is to develop the splitting extrapolation method to get a better approximation by computing a set of smaller discrete subproblems in parallel. We therefore need to establish the multiparameter asymptotic expansion of the fully discrete $d$-quadratic isoparametric finite element error and develop the splitting extrapolation formulas, which is the focus of this paper.

In the rest of this section, we list more conventions used in this article. Let $\|\cdot\|_{k, p, \hat{\Omega}}$ denote the norm of the space $W_{p}^{k}(\hat{\Omega}),\|\cdot\|_{k, \hat{\Omega}}$ denote the norm of the space $H^{k}(\hat{\Omega})$, and $\|\cdot\|_{k, \infty, \hat{\Omega}}$ denote the norm of the space $W_{\infty}^{k}(\hat{\Omega})$. We define $\prod_{s=1}^{m} W_{p}^{k}\left(\hat{\Omega}_{s}\right)$ to be a product space with the norm $\|\cdot\|_{k, p, \hat{\Omega}}^{\prime}:=\left(\sum_{s=1}^{m}\|\cdot\|_{k, p, \hat{\Omega}_{s}}^{p}\right)^{\frac{1}{p}}, \prod_{s=1}^{m} H^{k}\left(\hat{\Omega}_{s}\right)$ to be a product space with the norm $\|\cdot\|_{k, \hat{\Omega}}^{\prime}:=\left(\sum_{s=1}^{m}\|\cdot\|_{k, \hat{\Omega}_{s}}^{2}\right)^{\frac{1}{2}}$, and $\prod_{s=1}^{m} W_{\infty}^{k}\left(\hat{\Omega}_{s}\right)$ to be a product space with the norm $\|\cdot\|_{k, \infty, \hat{\Omega}}^{\prime}:=\sup _{1 \leq s \leq m}\|\cdot\|_{k, \infty, \hat{\Omega}_{s}} \cdot$ Define $\hat{h}:=\left(\hat{h}_{1}, \ldots, \hat{h}_{l+1}\right), \hat{h}_{0}:=\max _{1 \leq i \leq l} \hat{h}_{i}$.

3. Multiparameter asymptotic expansion of the semidiscrete finite element error. In section 2 we presented the semidiscrete finite element approximation (2.7). In this section we will show the multiparameter asymptotic expansion of its error. This is the key step in proving the multiparameter asymptotic expansion of the fully discrete finite element error in section 4.

First, we prove the following lemma, which is analogous to Lemma 1 in [12] and Lemma 13.1 in [26]. However, in this article we need to deal with the bilinear form $A(t ; \hat{u}, \hat{v})$, which has one more term $\hat{q} \hat{u} \hat{v}$, by using assumptions (2.3), (2.4), and (2.5).

LEMma 3.1. Along with the assumption of (2.3), (2.4), and (2.5), if $\hat{u}_{0} \in$ $H^{1}(\hat{\Omega}), \hat{u}_{1} \in L^{2}(\hat{\Omega}), \hat{f} \in L^{2}\left(\hat{Q}_{T}\right)$, then there exists a constant $C$ independent of $\hat{h}$, such that the solution of (2.7) satisfies

$$
\|\overline{\hat{u}}\|_{1, \hat{\Omega}}^{2}+\left\|\overline{\hat{u}}_{t}\right\|_{0, \hat{\Omega}}^{2} \leq C\left(\left\|\hat{u}_{0}\right\|_{1, \hat{\Omega}}^{2}+\left\|\hat{u}_{1}\right\|_{0, \hat{\Omega}}^{2}+\|\hat{f}\|_{0, \hat{Q}_{T}}^{2}\right) .
$$

Proof. Let $\hat{v}=\overline{\hat{u}}_{t}$ in (2.7); then because of the Leibniz integral rule

$$
\frac{d}{\mathrm{~d} t}\left(\overline{\hat{u}}_{t}, \overline{\hat{u}}_{t}\right)=2\left(\overline{\hat{u}}_{t t}, \overline{\hat{u}}_{t}\right) \text { and } \frac{d}{\mathrm{~d} t} A(t ; \overline{\hat{u}}, \overline{\hat{u}})=A^{\prime}(t ; \overline{\hat{u}}, \overline{\hat{u}})+2 A\left(t ; \overline{\hat{u}}, \overline{\hat{u}}_{t}\right)
$$

lead to

$$
\frac{d}{\mathrm{~d} s}\left(\overline{\hat{u}}_{s}, \overline{\hat{u}}_{s}\right)+\frac{d}{\mathrm{~d} s} A(s ; \overline{\hat{u}}, \overline{\hat{u}})-A^{\prime}(s ; \overline{\hat{u}}, \overline{\hat{u}})=2\left(\hat{f}, \overline{\hat{u}}_{s}\right) .
$$

In (3.2) we've written $s$ instead of $t$. Then integrating (3.2) from 0 to $t$ with respect to $s$ and using (2.8), we have

$$
\begin{aligned}
\left\|\overline{\hat{u}}_{t}\right\|_{0, \hat{\Omega}}^{2}+A(t ; \overline{\hat{u}}, \overline{\hat{u}})= & \left\|\hat{P}_{h} \hat{u}_{1}\right\|_{0, \hat{\Omega}}^{2}+A\left(0 ; \hat{P}_{h} \hat{u}_{0}, \hat{P}_{h} \hat{u}_{0}\right)+\int_{0}^{t} A^{\prime}(s ; \overline{\hat{u}}, \overline{\hat{u}}) \mathrm{d} s \\
& +\int_{0}^{t} \int_{\hat{\Omega}} 2 \hat{f} \hat{\hat{u}}_{s} \mathrm{~d} \hat{x} \mathrm{~d} s .
\end{aligned}
$$

Copyright $\odot$ by SIAM. Unauthorized reproduction of this article is prohibited. 
Because of (2.3), (2.4), and (2.5), we get

$$
\begin{aligned}
\left\|\overline{\hat{u}}_{t}\right\|_{0, \hat{\Omega}}^{2}+\mu_{1}\|\overline{\hat{u}}\|_{1, \hat{\Omega}}^{2} \leq & \left\|\hat{P}_{h} \hat{u}_{1}\right\|_{0, \hat{\Omega}}^{2}+\mu_{2}\left\|\hat{P}_{h} \hat{u}_{0}\right\|_{1, \hat{\Omega}}^{2}+\int_{0}^{t} \mu_{3}\|\overline{\hat{u}}\|_{1, \hat{\Omega}}^{2} \mathrm{~d} s+\|\hat{f}\|_{0, \hat{Q}_{T}}^{2} \\
& +\int_{0}^{t}\left\|\overline{\hat{u}}_{s}\right\|_{0, \hat{\Omega}}^{2} \mathrm{~d} s .
\end{aligned}
$$

Then there exist constants $C_{1}$ and $C_{2}$ such that

$$
\left.\left\|\overline{\hat{u}}_{t}\right\|_{0, \hat{\Omega}}^{2}+\|\overline{\hat{u}}\|_{1, \hat{\Omega}}^{2} \leq C_{1}\left(\left\|\hat{P}_{h} \hat{u}_{1}\right\|_{0, \hat{\Omega}}^{2}+\left\|\hat{P}_{h} \hat{u}_{0}\right\|_{1, \hat{\Omega}}^{2}+\|\hat{f}\|_{0, \hat{Q}_{T}}^{2}\right)+C_{2} \int_{0}^{t}\left(\left\|\overline{\hat{u}}_{s}\right\|_{0, \hat{\Omega}}^{2}+\|\overline{\hat{u}}\|_{1, \hat{\Omega}}^{2}\right) \mathrm{d} s\right) .
$$

With Gronwall's inequality and the boundedness of $\hat{P}_{h}$, we finish the proof.

Second, we recall the following two lemmas from $[18,38]$. Define $\hat{u}^{I}$ to be the interpolation function of $\hat{u}$ in $H^{2}\left(0, T ; \hat{S}_{0}^{h}\right)$. Let $\hat{R}_{h}^{t}$ denote the Ritz projection operator with respect to $A(t ; \cdot, \cdot)$, i.e., $A\left(t ; \hat{R}_{h}^{t} \hat{u}, \hat{v}\right)=A(t ; \hat{u}, \hat{v})$ for all $\hat{v} \in \hat{S}_{0}^{h}$.

LEMma 3.2. Assume that $\hat{a}_{i j}, \hat{q} \in\left(\prod_{k=1}^{m} W_{\infty}^{4}\left(\hat{\Omega}_{k}\right)\right) \bigcap L^{\infty}(\hat{\Omega})$, and $\hat{u}(t, \cdot) \in$ $\left(\prod_{k=1}^{m} H^{7}\left(\hat{\Omega}_{k}\right)\right) \cap H_{0}^{1}(\hat{\Omega})$. Then there exist an $r>0$ and functions $\hat{\phi}_{i} \in \prod_{k=1}^{m}\left(H^{r}\left(\hat{\Omega}_{k}\right)\right.$ $\left.\bigcap L^{\infty}\left(\hat{\Omega}_{k}\right)\right)(i=1, \ldots, l)$ independent of $\hat{h}$ such that

$$
\hat{R}_{h}^{t} \hat{u}-\hat{u}^{I}=\sum_{i=1}^{l} \hat{h}_{i}^{4} \hat{\phi}_{i}^{I}+\varepsilon,\|\varepsilon\|_{0, \infty, \hat{\Omega}}^{\prime}=O\left(\hat{h}_{0}^{4+\alpha}\left|\ln \hat{h}_{0}\right|^{\frac{d-1}{d}}\right), \alpha=\min (r, 2)-\frac{d}{2}>0 .
$$

Lemma 3.3. Given the same assumption as in Lemma 3.2, there exist an $r>0$ and functions $\hat{W}_{i} \in \prod_{k=1}^{m}\left(H^{r}\left(\hat{\Omega}_{k}\right) \bigcap L^{\infty}\left(\hat{\Omega}_{k}\right)\right)(i=1, \ldots, l)$ and a constant $C$ independent of $\hat{h}$ such that

$$
\left\|\hat{R}_{h}^{t} \hat{u}-\hat{P}_{h} \hat{u}-\sum_{i=1}^{l} \hat{h}_{i}^{4} \hat{P}_{h} \hat{W}_{i}\right\|_{0, \infty, \hat{\Omega}}^{\prime} \leq C \hat{h}_{0}^{4+\beta_{0}}, \beta_{0}=\min (r, 2)-\frac{d}{2}>0 .
$$

Based on the three lemmas above, we prove the following theorem, which shows the multiparameter asymptotic expansion of the semidiscrete $d$-quadratic isoparametric finite element approximation.

THEOREM 3.4. Along with the same assumptions of Lemma 3.2, there exist functions $\hat{\psi}_{i} \in H^{2}\left(0, T ; H^{1}(\hat{\Omega})\right)(i=1, \ldots, l)$ independent of $\hat{h}$ such that the error of the solution of (2.7) satisfies the following multiparameter asymptotic expansion:

$$
\overline{\hat{u}}-\hat{u}^{I}=\sum_{i=1}^{l} \hat{h}_{i}^{4} \hat{\psi}_{i}^{I}+\varepsilon,\|\varepsilon\|_{0, \infty, \hat{Q}_{T}}^{\prime}=O\left(\hat{h}_{0}^{4+\beta_{1}}\left|\ln \hat{h}_{0}\right|^{\frac{d-1}{d}}\right), \beta_{1}>0 .
$$

Proof. Let $\hat{\theta}=\hat{R}_{h}^{t} \hat{u}-\overline{\hat{u}}$; then

$$
\overline{\hat{u}}-\hat{u}^{I}=-\hat{\theta}+\left(\hat{R}_{h}^{t} \hat{u}-\hat{u}^{I}\right)
$$

By Lemma 3.2, there exists some $\alpha>0$ such that we have

$$
\hat{R}_{h}^{t} \hat{u}-\hat{u}^{I}=\sum_{i=1}^{l} \hat{h}_{i}^{4} \hat{\phi}_{i}^{I}+\varepsilon_{1},\left\|\varepsilon_{1}\right\|_{0, \infty, \hat{\Omega}}^{\prime}=O\left(\hat{h}_{0}^{4+\alpha}\left|\ln \hat{h}_{0}\right|^{\frac{d-1}{d}}\right) .
$$

Copyright (C) by SIAM. Unauthorized reproduction of this article is prohibited. 
Now we need to analyze the expansion of $\hat{\theta}$. Let $\hat{\rho}=\hat{u}-\hat{R}_{h}^{t} \hat{u}$. Then $\hat{P}_{h} \hat{\rho}=\hat{P}_{h} \hat{u}-\hat{R}_{h}^{t} \hat{u}$. From Lemma 3.3, we get

$$
\hat{P}_{h} \hat{\rho}=-\sum_{i=1}^{l} \hat{h}_{i}^{4} \hat{P}_{h} \hat{W}_{i}+\varepsilon_{2}, \quad \text { where }\left\|\varepsilon_{2}\right\|_{0, \infty, \hat{\Omega}}^{\prime}=O\left(\hat{h}_{0}^{4+\beta_{0}}\right) .
$$

Define $D_{t}^{m}=\frac{\partial^{m}}{\partial t^{m}}$. We note that $\hat{P}_{h}$ can commute with $D_{t}^{m}$. For any $\hat{v} \in \hat{S}_{0}^{h}$, using the definitions of $\hat{\theta}, \hat{\rho}, \hat{P}_{h}$, and $\hat{R}_{h}^{t}$ together with the formulas (2.6), (2.7), and (3.8), we have

$$
\begin{aligned}
& \left(\hat{\theta}_{t t}, \hat{v}\right)+A(t ; \hat{\theta}, \hat{v})=\left[\left(D_{t}^{2} \hat{R}_{h}^{t} \hat{u}, \hat{v}\right)+A\left(t ; \hat{R}_{h}^{t} \hat{u}, \hat{v}\right)\right]-\left[\left(\overline{\hat{u}}_{t t}, \hat{v}\right)+A(t ; \overline{\hat{u}}, \hat{v})\right] \\
& =\left[\left(D_{t}^{2} \hat{R}_{h}^{t} \hat{u}, \hat{v}\right)+A(t ; \hat{u}, \hat{v})\right]-(\hat{f}, \hat{v})=\left(D_{t}^{2} \hat{R}_{h}^{t} \hat{u}, \hat{v}\right)-\left(\hat{u}_{t t}, \hat{v}\right) \\
& =\left(D_{t}^{2} \hat{R}_{h}^{t} \hat{u}, \hat{v}\right)-\left(\hat{P}_{h} \hat{u}_{t t}, \hat{v}\right)=\left(D_{t}^{2} \hat{R}_{h}^{t} \hat{u}, \hat{v}\right)-\left(D_{t}^{2} \hat{P}_{h} \hat{u}, \hat{v}\right) \\
& =-\left(D_{t}^{2} \hat{P}_{h} \hat{\rho}, \hat{v}\right) \\
& =\sum_{i=1}^{l} \hat{h}_{i}^{4}\left(D_{t}^{2} \hat{P}_{h} \hat{W}_{i}, \hat{v}\right)-\left(D_{t}^{2} \varepsilon_{2}, \hat{v}\right), \\
& (\hat{\theta}(0, \cdot), \hat{v})=\left(\hat{R}_{h}^{t} \hat{u}(0, \cdot)-\overline{\hat{u}}(0, \cdot), \hat{v}\right)=\left(\hat{R}_{h}^{t} \hat{u}_{0}-\hat{u}_{0}, \hat{v}\right)=\left(\hat{P}_{h} \rho(0, \cdot), \hat{v}\right) \\
& =\sum_{i=1}^{l} \hat{h}_{i}^{4}\left(\hat{P}_{h} \hat{W}_{i}(0, \cdot), \hat{v}\right)-\left(\varepsilon_{2}(0, \cdot), \hat{v}\right), \\
& \left(D_{t} \hat{\theta}(0, \cdot), \hat{v}\right)=\sum_{i=1}^{l} \hat{h}_{i}^{4}\left(D_{t} \hat{P}_{h} \hat{W}_{i}(0, \cdot), \hat{v}\right)-\left(D_{t} \varepsilon_{2}(0, \cdot), \hat{v}\right)
\end{aligned}
$$

An auxiliary problem is constructed as follows: Find $\hat{\varphi}_{i} \in H^{2}\left(0, T ; H_{0}^{1}(\hat{\Omega})\right), i=$ $1, \ldots, l$, such that

$$
\left\{\begin{array}{l}
\left(D_{t}^{2} \hat{\varphi}_{i}, \hat{v}\right)+A\left(t ; \hat{\varphi}_{i}, \hat{v}\right)=-\left(D_{t}^{2} \hat{P}_{h} \hat{W}_{i}, \hat{v}\right) \\
\hat{\varphi}_{i}(0, \cdot)=-\hat{W}_{i}(0, \cdot), D_{t} \hat{\varphi}_{i}(0, \cdot)=-D_{t} \hat{W}_{i}(0, \cdot) .
\end{array} \quad \forall \hat{v} \in H_{0}^{1}(\hat{\Omega})\right.
$$

The semidiscrete finite element approximation can be obtained as follows: Find $\overline{\hat{\varphi}}_{i} \in$ $H^{2}\left(0, T ; \hat{S}_{0}^{h}\right), i=1, \ldots, l$, such that

$$
\begin{cases}\left(D_{t}^{2} \overline{\hat{\varphi}}_{i}, \hat{v}\right)+A\left(t ; \overline{\hat{\varphi}}_{i}, \hat{v}\right)=-\left(D_{t}^{2} \hat{P}_{h} \hat{W}_{i}, \hat{v}\right) & \forall \hat{v} \in \hat{S}_{0}^{h}, \\ \left(\hat{\bar{\varphi}}_{i}(0, \cdot), \hat{v}\right)=-\left(\hat{W}_{i}(0, \cdot), \hat{v}\right),\left(D_{t} \overline{\hat{\varphi}}_{i}(0, \cdot), \hat{v}\right)=-\left(D_{t} \hat{W}_{i}(0, \cdot), \hat{v}\right) & \forall \hat{v} \in \hat{S}_{0}^{h}\end{cases}
$$

Define $\overline{\hat{\psi}}=\hat{\theta}+\sum_{i=1}^{l} \hat{h}_{i}^{4} \overline{\hat{\varphi}}_{i}$; then (3.9), (3.10), (3.11), and (3.12) lead to

$$
\begin{cases}\left(D_{t}^{2} \overline{\hat{\psi}}, \hat{v}\right)+A(t ; \overline{\hat{\psi}}, \hat{v})=-\left(D_{t}^{2} \varepsilon_{2}, \hat{v}\right) & \forall \hat{v} \in \hat{S}_{0}^{h} \\ (\overline{\hat{\psi}}(0, \cdot), \hat{v})=-\left(\varepsilon_{2}(0, \cdot), \hat{v}\right),\left(D_{t}, \hat{\bar{\psi}}(0, \cdot), \hat{v}\right)=-\left(D_{t} \varepsilon_{2}(0, \cdot), \hat{v}\right) & \forall \hat{v} \in \hat{S}_{0}^{h} .\end{cases}
$$

Applying Lemma 3.1 to (3.13), we have

$$
\left\|\overline{\hat{\psi}}_{t}\right\|_{0, \hat{\Omega}}+\|\overline{\hat{\psi}}\|_{1, \hat{\Omega}} \leq C\left(\left\|\varepsilon_{2}(0)\right\|_{1, \hat{\Omega}}+\left\|D_{t} \varepsilon_{2}(0)\right\|_{0, \hat{\Omega}}+\left\|D_{t}^{2} \varepsilon_{2}\right\|_{0, \hat{Q}_{T}}\right) .
$$

Using (3.8), (3.14), and the inverse estimate [10], we can get

$$
\|\overline{\hat{\psi}}\|_{0, \infty, \hat{\Omega}} \leq C(t) \hat{h}_{0}^{4+\beta_{0}}\left|\ln \hat{h}_{0}\right|^{\frac{d-1}{d}} .
$$

Copyright $@$ by SIAM. Unauthorized reproduction of this article is prohibited. 
Note that $d=2,3$ in (2.1). If $1 \geq \gamma>\frac{d}{2}-1$ and $\hat{\varphi}_{i}(t, \cdot) \in H^{2}\left(0, T ; \prod_{k=1}^{m} H^{1+\gamma}\left(\hat{\Omega}_{k}\right) \bigcap\right.$ $\left.H_{0}^{1}(\hat{\Omega})\right)$, then by Theorem 4.2 in [2] or by the conclusions in $[12,26]$, we have

$$
\left\|\hat{\varphi}_{i}-\overline{\hat{\varphi}}_{i}\right\|_{1, \hat{\Omega}} \leq C \hat{h}_{0}^{\gamma}
$$

Replacing $\overline{\hat{\varphi}}_{i}$ by $\hat{\varphi}_{i}^{I}$ in the definition of $\overline{\hat{\psi}}$ and using (3.15), (3.16), and the inverse estimate, we get

$$
\left\|\hat{\theta}+\sum_{i=1}^{l} \hat{h}_{i}^{4} \hat{\varphi}_{i}^{I}\right\|_{0, \infty, \hat{\Omega}} \leq C\left|\ln \hat{h}_{0}\right|^{\frac{d-1}{d}} \hat{h}_{0}^{4+\beta_{2}}, \beta_{2}=\min \left(\beta_{0}, \gamma+1-\frac{d}{2}\right)>0 .
$$

Therefore,

$$
\hat{\theta}=-\sum_{i=1}^{l} \hat{h}_{i}^{4} \hat{\varphi}_{i}^{I}+\varepsilon_{3}, \text { where }\left\|\varepsilon_{3}\right\|_{0, \infty, \hat{\Omega}} \leq C\left|\ln \hat{h}_{0}\right|^{\frac{d-1}{d}} \hat{h}_{0}^{4+\beta_{2}}
$$

Plugging (3.7) and (3.17) into (3.6), we get

$$
\overline{\hat{u}}-\hat{u}^{I}=\sum_{i=1}^{l} \hat{h}_{i}^{4}\left(\hat{\varphi}_{i}^{I}+\hat{\phi}_{i}^{I}\right)+\varepsilon_{2}-\varepsilon_{3}
$$

Let $\hat{\psi}_{i}=\hat{\varphi}_{i}+\hat{\phi}_{i}$ and $\varepsilon=\varepsilon_{2}-\varepsilon_{3}$; then $\hat{\psi}_{i}^{I}=\hat{\varphi}_{i}^{I}+\hat{\phi}_{i}^{I}$ and

$$
\overline{\hat{u}}-\hat{u}^{I}=\sum_{i=1}^{l} \hat{h}_{i}^{4} \hat{\psi}_{i}^{I}+\varepsilon
$$

Finally, (3.17), (3.7), and (3.18) lead to

$$
\|\varepsilon\|_{0, \infty, \hat{Q}_{T}}^{\prime}=O\left(\hat{h}_{0}^{4+\beta_{1}}\left|\ln \hat{h}_{0}\right|^{\frac{d-1}{d}}\right), \beta_{1}=\min \left(\beta_{2}, \alpha\right)>0 .
$$

4. Multiparameter asymptotic expansion of the fully discrete finite element error. We presented the fully discrete finite element approximation (2.9) in section 2 and showed the multiparameter asymptotic expansion of the semidiscrete finite element error in section 3. Based on these results, we will establish the multiparameter asymptotic expansion of the fully discrete finite element error in this section.

Similar to [12], we introduce some finite difference operators as follows:

$$
\hat{U}^{n, \frac{1}{4}}=\frac{\hat{U}^{n+1}+2 \hat{U}^{n}+\hat{U}^{n-1}}{4}, \quad \hat{U}^{n+\frac{1}{2}}=\frac{\hat{U}^{n+1}+\hat{U}^{n}}{2}, \quad \hat{U}^{n-\frac{1}{2}}=\frac{\hat{U}^{n-1}+\hat{U}^{n}}{2},
$$

$$
\bar{\partial}_{t} \hat{U}^{n+\frac{1}{2}}=\frac{\hat{U}^{n+1}-\hat{U}^{n}}{\tau}, \quad \bar{\partial}_{t} \hat{U}^{n}=\frac{\hat{U}^{n+\frac{1}{2}}-\hat{U}^{n-\frac{1}{2}}}{\tau}, \quad \bar{\partial}_{t t} \hat{U}^{n}=\frac{\hat{U}^{n+1}-2 \hat{U}^{n}+\hat{U}^{n-1}}{\tau^{2}} .
$$


Then we have the following relations:

$$
\begin{aligned}
& \bar{\partial}_{t} \hat{U}^{n-\frac{1}{2}}=\bar{\partial}_{t} \hat{U}^{n-1+\frac{1}{2}}=\frac{\hat{U}^{n}-\hat{U}^{n-1}}{\tau}, \\
& \bar{\partial}_{t} \hat{U}^{n}=\frac{\hat{U}^{n+\frac{1}{2}}-\hat{U}^{n-\frac{1}{2}}}{\tau}=\frac{\bar{\partial}_{t} \hat{U}^{n+\frac{1}{2}}+\bar{\partial}_{t} \hat{U}^{n-\frac{1}{2}}}{2}, \\
& \bar{\partial}_{t t} \hat{U}^{n}=\frac{\hat{U}^{n+1}-2 \hat{U}^{n}+\hat{U}^{n-1}}{\tau^{2}}=\frac{\bar{\partial}_{t} \hat{U}^{n+\frac{1}{2}}-\bar{\partial}_{t} \hat{U}^{n-\frac{1}{2}}}{\tau}, \\
& \hat{U}^{n, \frac{1}{4}}=\frac{\hat{U}^{n+1}+2 \hat{U}^{n}+\hat{U}^{n-1}}{4}=\frac{\hat{U}^{n+\frac{1}{2}}+\hat{U}^{n-\frac{1}{2}}}{2} .
\end{aligned}
$$

The following lemma is analogous to Lemma 6 in [12], Lemma 13.2 in [26], and Lemma 3.1 in section 3 .

Lemma 4.1. Suppose $\hat{f} \in C\left(0, T ; L^{2}(\hat{\Omega})\right)$. Then there exists a constant $C$ independent of $\tau$ such that

$$
\max _{1 \leq n \leq N}\left(\left\|\bar{\partial}_{t} \hat{U}^{n-\frac{1}{2}}\right\|_{0, \hat{\Omega}}^{2}+\left\|\hat{U}^{n-\frac{1}{2}}\right\|_{1, \hat{\Omega}}^{2}\right) \leq C\left(\left\|\hat{U}^{\frac{1}{2}}\right\|_{1, \hat{\Omega}}^{2}+\left\|\bar{\partial}_{t} \hat{U}^{\frac{1}{2}}\right\|_{0, \hat{\Omega}}^{2}+\max _{1 \leq n \leq N}\|\hat{f}\|_{0, \hat{\Omega}}^{2}\right) .
$$

Proof. In (2.9); let $\hat{v}=\bar{\partial}_{t} \hat{U}^{n}$, then (4.4), (4.5), and (4.6) lead to

$$
\begin{aligned}
& \left\|\bar{\partial}_{t} \hat{U}^{n+\frac{1}{2}}\right\|_{0, \hat{\Omega}}^{2}-\left\|\bar{\partial}_{t} \hat{U}^{n-\frac{1}{2}}\right\|_{0, \hat{\Omega}}^{2}+A\left(t_{n} ; \hat{U}^{n+\frac{1}{2}}, \hat{U}^{n+\frac{1}{2}}\right)-A\left(t_{n} ; \hat{U}^{n-\frac{1}{2}}, \hat{U}^{n-\frac{1}{2}}\right) \\
(4.8)= & \tau\left(\hat{f}^{n}, \bar{\partial}_{t} \hat{U}^{n+\frac{1}{2}}+\bar{\partial}_{t} \hat{U}^{n-\frac{1}{2}}\right) .
\end{aligned}
$$

By Taylor expansion, there exists a $\theta_{n} \in(0,1)$ such that

$$
A\left(t_{n} ; \hat{U}^{n-\frac{1}{2}}, \hat{U}^{n-\frac{1}{2}}\right)=A\left(t_{n-1} ; \hat{U}^{n-\frac{1}{2}}, \hat{U}^{n-\frac{1}{2}}\right)+\tau A^{\prime}\left(t_{n-\theta_{n}} ; \hat{U}^{n-\frac{1}{2}}, \hat{U}^{n-\frac{1}{2}}\right) .
$$

Here $t_{n-\theta_{n}}=t_{n}-\theta_{n} \tau$. Plugging (4.9) into (4.8) and taking the summation for $n=1, \ldots, N-1$ on both sides of (4.8), we get

$$
\begin{aligned}
& \left\|\bar{\partial}_{t} \hat{U}^{N-\frac{1}{2}}\right\|_{0, \hat{\Omega}}^{2}+A\left(t_{N-1} ; \hat{U}^{N-\frac{1}{2}}, \hat{U}^{N-\frac{1}{2}}\right) \\
= & \left\|\bar{\partial}_{t} \hat{U}^{\frac{1}{2}}\right\|_{0, \hat{\Omega}}^{2}+A\left(t_{0} ; \hat{U}^{\frac{1}{2}}, \hat{U}^{\frac{1}{2}}\right)+\tau \sum_{n=1}^{N-1} A^{\prime}\left(t_{n-\theta_{n}} ; \hat{U}^{n-\frac{1}{2}}, \hat{U}^{n-\frac{1}{2}}\right) \\
& +\tau \sum_{n=1}^{N-1}\left(\hat{f}^{n}, \bar{\partial}_{t} \hat{U}^{n+\frac{1}{2}}+\bar{\partial}_{t} \hat{U}^{n-\frac{1}{2}}\right) .
\end{aligned}
$$

Copyright $\odot$ by SIAM. Unauthorized reproduction of this article is prohibited. 
Using (2.3), (2.4), (2.5), (4.2), (4.3), and the Schwarz inequality, we have

$$
\begin{aligned}
& \left\|\bar{\partial}_{t} \hat{U}^{N-\frac{1}{2}}\right\|_{0, \hat{\Omega}}^{2}+\mu_{1}\left\|\hat{U}^{N-\frac{1}{2}}\right\|_{1, \hat{\Omega}}^{2} \\
\leq & \left\|\bar{\partial}_{t} \hat{U}^{\frac{1}{2}}\right\|_{0, \hat{\Omega}}^{2}+\mu_{2}\left\|\hat{U}^{\frac{1}{2}}\right\|_{1, \hat{\Omega}}^{2}+\tau \sum_{n=1}^{N-1} \mu_{3}\left\|\hat{U}^{n-\frac{1}{2}}\right\|_{1, \hat{\Omega}}^{2} \\
& +\tau \sum_{n=1}^{N-1}\left(\left\|\hat{f}^{n}\right\|_{0, \hat{\Omega}}\left\|\bar{\partial}_{t} \hat{U}^{n+\frac{1}{2}}\right\|_{0, \hat{\Omega}}+\left\|\hat{f}^{n}\right\|_{0, \hat{\Omega}}\left\|\bar{\partial}_{t} \hat{U}^{n-\frac{1}{2}}\right\|_{0, \hat{\Omega}}\right) \\
\leq & \left\|\bar{\partial}_{t} \hat{U}^{\frac{1}{2}}\right\|_{0, \hat{\Omega}}^{2}+\mu_{2}\left\|\hat{U}^{\frac{1}{2}}\right\|_{1, \hat{\Omega}}^{2}+\tau \sum_{n=1}^{N-1} \mu_{3}\left\|\hat{U}^{n-\frac{1}{2}}\right\|_{1, \hat{\Omega}}^{2} \\
& +\frac{\tau}{2} \sum_{n=1}^{N-1}\left(2\left\|\hat{f}^{n}\right\|_{0, \hat{\Omega}}^{2}+\left\|\bar{\partial}_{t} \hat{U}^{n+\frac{1}{2}}\right\|_{0, \hat{\Omega}}^{2}+\left\|\bar{\partial}_{t} \hat{U}^{n-\frac{1}{2}}\right\|_{0, \hat{\Omega}}^{2}\right) \\
\leq & \left\|\bar{\partial}_{t} \hat{U}^{\frac{1}{2}}\right\|_{0, \hat{\Omega}}^{2}+\mu_{2}\left\|\hat{U}^{\frac{1}{2}}\right\|_{1, \hat{\Omega}}^{2}+\tau \sum_{n=1}^{N-1} \mu_{3}\left\|\hat{U}^{n-\frac{1}{2}}\right\|_{1, \hat{\Omega}}^{2}+T \max _{0 \leq t \leq T}\|\hat{f}\|_{0, \hat{\Omega}}^{2}+\tau \sum_{n=1}^{N-1}\left\|\bar{\partial}_{t} \hat{U}^{n-\frac{1}{2}}\right\|_{0, \hat{\Omega}}^{2} \\
& +\frac{\tau}{2}\left\|\bar{\partial}_{t} \hat{U}^{N-\frac{1}{2}}\right\|_{0, \hat{\Omega}}^{2} .
\end{aligned}
$$

Therefore, by moving $\frac{\tau}{2}\left\|\bar{\partial}_{t} \hat{U}^{N-\frac{1}{2}}\right\|_{0, \hat{\Omega}}^{2}$ to the left-hand side and assuming $\tau$ is sufficiently small, there exist constants $C_{1}$ and $C_{2}$ independent of $\tau$ such that

$$
\begin{aligned}
& \left\|\bar{\partial}_{t} \hat{U}^{N-\frac{1}{2}}\right\|_{0, \hat{\Omega}}^{2}+\left\|\hat{U}^{N-\frac{1}{2}}\right\|_{1, \hat{\Omega}}^{2} \\
\leq & C_{1}\left(\left\|\hat{U}^{\frac{1}{2}}\right\|_{1, \hat{\Omega}}^{2}+\left\|\bar{\partial}_{t} \hat{U}^{\frac{1}{2}}\right\|_{0, \hat{\Omega}}^{2}+\max _{0 \leq t \leq T}\|\hat{f}\|_{0, \hat{\Omega}}^{2}\right)+C_{2} \tau \sum_{n=1}^{N-1}\left(\left\|\bar{\partial}_{t} \hat{U}^{n-\frac{1}{2}}\right\|_{0, \hat{\Omega}}^{2}+\left\|\hat{U}^{n-\frac{1}{2}}\right\|_{1, \hat{\Omega}}^{2}\right) .
\end{aligned}
$$

With the discrete Gronwall inequality, we finish the proof.

Note that the finite difference scheme in $(2.9)$ has $O\left(\tau^{2}\right)$ order of accuracy. Therefore, based on the lemma above and the Taylor expansion, we can prove the following lemma. Let $\hat{\Omega}_{0}^{h}$ denote the set of grid points obtained from the initial grid parameter $\hat{h}=\left(\hat{h}_{1}, \ldots, \hat{h}_{l+1}\right)$.

Lemma 4.2. Assume that the solution of (2.7) satisfies $\overline{\hat{u}} \in C^{5}\left(0, T ; \hat{S}_{0}^{h}\right)$. Then there exists a function $\hat{\psi}_{l+1} \in H^{2}\left(0, T ; \hat{S}_{0}^{h}\right)$ independent of $\hat{h}$ such that

$$
\begin{aligned}
& \hat{U}^{n}(\hat{X})-\overline{\hat{u}}^{n}(\hat{X})=\tau^{2} \hat{\psi}_{l+1}^{n}(\hat{X})+\overline{\hat{r}}^{n}(\hat{X}) \quad \forall \hat{X} \in \hat{\Omega}_{0}^{h}, 1 \leq n \leq N, \\
& \max _{1 \leq n \leq N}\left(\left\|\partial_{t} \overline{\hat{r}}^{n-\frac{1}{2}}\right\|_{0, \hat{\Omega}}+\left\|\overline{\hat{r}}^{n-\frac{1}{2}}\right\|_{1, \hat{\Omega}}\right) \leq C \tau^{3} .
\end{aligned}
$$

Proof. We use the method of undetermined coefficients to prove this lemma. Assume

$$
\hat{U}^{n}-\overline{\hat{u}}^{n}=\tau^{2} \hat{\psi}_{l+1}^{n}+\overline{\hat{r}}^{n},
$$

where $\hat{\psi}_{l+1}^{n}$ and $\overline{\hat{r}}^{n}$ are undetermined. By the Taylor expansions of $\overline{\hat{u}}^{n+1}$ and $\overline{\hat{u}}^{n-1}$ at $n \tau$, we have

$$
\overline{\hat{u}}^{n, \frac{1}{4}}=\frac{\overline{\hat{u}}^{n+1}+2 \overline{\hat{u}}^{n}+\overline{\hat{u}}^{n-1}}{4}=\overline{\hat{u}}^{n}+\frac{1}{4} \tau^{2} D_{t}^{2} \overline{\hat{u}}^{n}+\varepsilon_{1}^{n}, \quad \varepsilon_{1}^{n}=\frac{\tau^{4}}{96}\left(D_{t}^{4} \overline{\hat{u}}\left(\theta_{1}, \hat{x}\right)+D_{t}^{4} \overline{\hat{u}}\left(\theta_{2}, \hat{x}\right)\right),
$$

Copyright (c) by SIAM. Unauthorized reproduction of this article is prohibited. 
where $(n-1) \tau \leq \theta_{1} \leq n \tau$ and $n \tau \leq \theta_{2} \leq(n+1) \tau$. Then (4.1), (4.12), and (4.13) lead to

$$
\hat{U}^{n, \frac{1}{4}}=\overline{\hat{u}}^{n, \frac{1}{4}}+\tau^{2} \hat{\psi}_{l+1}^{n, \frac{1}{4}}+\overline{\hat{r}}^{n, \frac{1}{4}}=\overline{\hat{u}}^{n}+\frac{1}{4} \tau^{2} D_{t}^{2} \overline{\hat{u}}^{n}+\tau^{2} \hat{\psi}_{l+1}^{n, \frac{1}{4}}+\overline{\hat{r}}^{n, \frac{1}{4}}+\varepsilon_{1}^{n} .
$$

Similarly, by the Taylor expansions of $\overline{\hat{u}}^{n+1}$ and $\overline{\hat{u}}^{n-1}$ at $n \tau$, we get

$$
\begin{aligned}
\bar{\partial}_{t t} \overline{\hat{u}}^{n} & =\frac{\overline{\hat{u}}^{n+1}-2 \overline{\hat{u}}^{n}+\overline{\hat{u}}^{n-1}}{\tau^{2}}=\overline{\hat{u}}_{t t}^{n}+\frac{1}{12} \tau^{2} D_{t}^{4} \overline{\hat{u}}^{n}+\varepsilon_{2}^{n}, \\
\varepsilon_{2}^{n} & =\frac{\tau^{3}}{120}\left(-D_{t}^{5} \overline{\hat{u}}\left(\theta_{3}, \hat{x}\right)+D_{t}^{5} \overline{\hat{u}}\left(\theta_{4}, \hat{x}\right)\right),
\end{aligned}
$$

where $(n-1) \tau \leq \theta_{3} \leq n \tau$ and $n \tau \leq \theta_{4} \leq(n+1) \tau$. Then by (4.2), (4.12), and (4.15), we have

$$
\bar{\partial}_{t t} \hat{U}^{n}=\bar{\partial}_{t t} \overline{\hat{u}}^{n}+\tau^{2} \bar{\partial}_{t t} \hat{\psi}_{l+1}^{n}+\bar{\partial}_{t t} \overline{\hat{r}}^{n}=\overline{\hat{u}}_{t t}^{n}+\frac{1}{12} \tau^{2} D_{t}^{4} \overline{\hat{u}}^{n}+\tau^{2} \bar{\partial}_{t t} \hat{\psi}_{l+1}^{n}+\bar{\partial}_{t t} \overline{\hat{r}}^{n}+\varepsilon_{2}^{n} .
$$

Hence (2.7), (2.9), (4.14) and (4.16) lead to

$$
\begin{aligned}
& \left(\frac{1}{12} \tau^{2} D_{t}^{4} \overline{\hat{u}}^{n}+\tau^{2} \bar{\partial}_{t t} \hat{\psi}_{l+1}^{n}+\bar{\partial}_{t t} \overline{\hat{r}}^{n}+\varepsilon_{2}^{n}, \hat{v}\right) \\
& +A\left(t_{n} ; \frac{1}{4} \tau^{2} D_{t}^{2} \overline{\hat{u}}^{n}+\tau^{2} \hat{\psi}_{l+1}^{n, \frac{1}{4}}+\overline{\hat{r}}^{n, \frac{1}{4}}+\varepsilon_{1}^{n}, \hat{v}\right)=0 \quad \forall \hat{v} \in \hat{S}_{0}^{h} .
\end{aligned}
$$

We choose $\hat{\psi}_{l+1} \in H^{2}\left(0, T ; \hat{S}_{0}^{h}\right)$ such that

$$
\left\{\begin{array}{l}
\left(\bar{\partial}_{t t} \hat{\psi}_{l+1}^{n}, \hat{v}\right)+A\left(t_{n} ; \hat{\psi}_{l+1}^{n, \frac{1}{4}}, \hat{v}\right)=-\frac{1}{12}\left(D_{t}^{4} \overline{\hat{u}}^{n}, \hat{v}\right)-\frac{1}{4} A\left(t_{n} ; D_{t}^{2} \overline{\hat{u}}^{n}, \hat{v}\right) \quad \forall \hat{v} \in \hat{S}_{0}^{h} \\
\hat{\psi}_{l+1}^{0}=0, \hat{\psi}_{l+1}^{1}=0
\end{array}\right.
$$

Using Taylor expansion, (2.7), and (2.9), we have

$$
\begin{aligned}
\overline{\hat{u}}^{1} & =\overline{\hat{u}}(\tau, \hat{x})=\overline{\hat{u}}(0, \hat{x})+\tau \overline{\hat{u}}_{t}(0, \hat{x})+\frac{\tau^{2}}{2} \overline{\hat{u}}_{t t}(0, \hat{x})+\frac{\tau^{3}}{6} D_{t}^{3} \overline{\hat{u}}(0, \hat{x})+\frac{\tau^{4}}{24} D_{t}^{4} \overline{\hat{u}}\left(\theta_{5}, \hat{x}\right) \\
(4.19) & =\hat{U}^{1}+\frac{\tau^{4}}{24} D_{t}^{4} \overline{\hat{u}}\left(\theta_{5}, \hat{x}\right),
\end{aligned}
$$

where $0 \leq \theta_{5} \leq \tau$. By (2.7), (2.9), (4.12), (4.17), (4.18), and (4.19), we get

$$
\left\{\begin{array}{l}
\left(\bar{\partial}_{t t} \overline{\hat{r}}^{n}, \hat{v}\right)+A\left(t_{n} ; \overline{\hat{r}}^{n, \frac{1}{4}}, \hat{v}\right)=-\left(\varepsilon_{2}^{n}, \hat{v}\right)-A\left(t_{n} ; \varepsilon_{1}^{n}, \hat{v}\right) \quad \forall \hat{v} \in \hat{S}_{0}^{h}, \\
\overline{\hat{r}}^{0}=0, \overline{\hat{r}}^{1}=-\frac{\tau^{4}}{24} D_{t}^{4} \overline{\hat{u}}\left(\theta_{5}, \hat{x}\right) .
\end{array}\right.
$$

Repeating the proof of Lemma 4.1, we get

$$
\begin{aligned}
& \max _{1 \leq n \leq N}\left(\left\|\partial_{t} \overline{\hat{r}}^{n-\frac{1}{2}}\right\|_{0, \hat{\Omega}}+\left\|\overline{\hat{r}}^{n-\frac{1}{2}}\right\|_{1, \hat{\Omega}}\right) \\
& \quad \leq C\left(\left\|\bar{\partial}_{t} \bar{r}^{\frac{1}{2}}\right\|_{0, \hat{\Omega}}+\left\|\bar{r}^{\frac{1}{2}}\right\|_{1, \hat{\Omega}}+\max _{1 \leq n \leq N}\left(\left\|\varepsilon_{2}^{n}\right\|_{0, \hat{\Omega}}+\left\|\varepsilon_{1}^{n}\right\|_{1, \hat{\Omega}}\right)\right) .
\end{aligned}
$$

Copyright (c) by SIAM. Unauthorized reproduction of this article is prohibited. 
Note (4.1), (4.2), and (4.20); thus

$$
\left\|\overline{\hat{r}}^{\frac{1}{2}}\right\|_{1, \hat{\Omega}} \leq C \tau^{4},\left\|\bar{\partial}_{t} \bar{r}^{\frac{1}{2}}\right\|_{0, \hat{\Omega}} \leq C \tau^{3} .
$$

Plugging (4.13), (4.15), and (4.22) into (4.21), we finish the proof.

The combination of the previous lemma and Theorem 3.4 gives us the main theorem of the paper, which shows the multiparameter asymptotic expansion of the fully discrete $d$-quadratic isoparametric finite element error.

TheOrem 4.3. Along with the same assumption of Lemma 4.2 and Theorem 3.4, there exist functions $\hat{\psi}_{i}(t, x)(i=1, \ldots, l+1)$ independent of $\hat{h}$ such that

$$
\begin{aligned}
\hat{U}^{n}(\hat{X})-\hat{u}^{n}(\hat{X}) & =\sum_{i=1}^{l} \hat{h}_{i}^{4} \hat{\psi}_{i}^{n}(\hat{X})+\hat{h}_{l+1}^{2} \hat{\psi}_{l+1}^{n}(\hat{X})+\varepsilon^{n} \quad \forall \hat{X} \in \hat{\Omega}_{0}^{h}, 1 \leq n \leq N, \\
24) \quad\left\|\varepsilon^{n}\right\|_{0, \infty, \hat{Q}_{T}}^{\prime} & =O\left(\hat{h}_{0}^{4+\beta_{1}}\left|\ln \hat{h}_{0}\right|^{\frac{d-1}{d}}+\hat{h}_{l+1}^{4-\frac{d}{2}}\left|\ln \hat{h}_{l+1}\right|^{\frac{d-1}{d}}\right), \hat{h}_{l+1}=\tau .
\end{aligned}
$$

Proof. Since $\hat{u}^{n}(\hat{X})=\hat{u}^{n I}(\hat{X})$ for all $\hat{X} \in \hat{\Omega}_{0}^{h}, 1 \leq n \leq N$, from Theorem 3.4 and Lemma 4.2, we get

$$
\begin{aligned}
\hat{U}^{n}(\hat{X})-\hat{u}^{n}(\hat{X}) & =\left(\hat{U}^{n}(\hat{X})-\overline{\hat{u}}^{n}(\hat{X})\right)+\left(\overline{\hat{u}}^{n}(\hat{X})-\hat{u}^{n}(\hat{X})\right) \\
& =\sum_{i=1}^{l} \hat{h}_{i}^{4} \hat{\psi}_{i}^{n}(\hat{X})+\hat{h}_{l+1}^{2} \hat{\psi}_{l+1}^{n}(\hat{X})+\varepsilon^{n} .
\end{aligned}
$$

Recalling (3.5) and (4.11), we get the estimate for $\varepsilon^{n}$.

Remark 4.1. Similar to Remark 4 in [38], the expansion holds not only for all the grid nodes in $\hat{\Omega}_{0}^{h}$ but also for all the edge midpoints and element centers in $\hat{\Omega}_{0}^{h}$.

Remark 4.2. From the above lemmas and theorems, we can see that the multiparameter expansion requires only the local smoothness of the solutions, i.e., the smoothness of the solutions in each subdomain $\hat{\Omega}_{k}, k=1, \ldots, m$. Therefore, splitting extrapolation is efficient for solving discontinuous problems if we regard the interfaces of the problem as the interfaces of the initial domain decomposition.

5. Splitting extrapolation formulas on the globally fine grid. In section 4 we established the multiparameter asymptotic expansion of the fully discrete finite element error. Based on this expansion, in this section we will develop the splitting extrapolation formulas by applying the basic idea of splitting extrapolation mentioned in section 1. We will develop the splitting extrapolation formulas for all the nodes in the globally fine grid, not only on the coarse grid and locally fine grids. Here the coarse grid is obtained from initial grid parameter $\hat{h}=\left(\hat{h}_{1}, \ldots, \hat{h}_{l+1}\right)$, the locally fine grid is obtained from $\hat{h}^{(i)}=\left(\hat{h}_{1}, \ldots, \frac{\hat{h}_{i}}{2}, \ldots, \hat{h}_{l+1}\right), i=1, \ldots, l+1$, and the globally fine grid is obtained from $\frac{\hat{h}}{2}$.

The following two lemmas use ideas similar to those presented in $[18,28,37,38$, 40]. Those references use similar conclusions to construct the splitting extrapolation formulas, but proofs are not given. Since the proofs give insight to the construction of splitting extrapolation formulas, we show the proofs here. Based on the two lemmas, we will construct the splitting extrapolation formulas for all the four types of grid points of the globally fine grid as follows.

Let $\varepsilon$ denote $O\left(\hat{h}_{0}^{4+\beta_{1}}\left|\ln \hat{h}_{0}\right|^{\frac{d-1}{d}}+\hat{h}_{l+1}^{4-\frac{d}{2}}\left|\ln \hat{h}_{l+1}\right|^{\frac{d-1}{d}}\right), \hat{\Omega}_{i}^{h}$ denote the set of grid points obtained from $\hat{h}^{(i)}=\left(\hat{h}_{1}, \ldots, \frac{\hat{h}_{i}}{2}, \ldots, \hat{h}_{l+1}\right), i=1, \ldots, l+1, \hat{U}_{0}^{n}$ denote the fully 
discrete approximation at time $t_{n}$ on $\hat{\Omega}_{0}^{h}$, and $\hat{U}_{i}^{n}$ denote the fully discrete approximation at time $t_{n}$ on $\hat{\Omega}_{i}^{h}, i=1, \ldots, l+1$.

(1) Type 0: grid points in $\hat{\Omega}_{0}^{h}$. Suppose $E$ is a grid point in $\hat{\Omega}_{0}^{h}$.

LEMMA 5.1.

$$
\frac{16}{15} \sum_{i=1}^{l} \hat{U}_{i}^{n}(E)+\frac{4}{3} \hat{U}_{(l+1)}^{n}(E)+\left[-\frac{16}{15} l-\frac{1}{3}\right] \hat{U}_{0}^{n}(E)-\hat{u}^{n}(E)=\varepsilon .
$$

Proof. From (4.23), we have

$$
\begin{aligned}
\hat{U}_{0}^{n}(E) & =\hat{u}^{n}(E)+\sum_{i=1}^{l} \hat{h}_{i}^{4} \hat{\psi}_{i}^{n}(E)+\hat{h}_{l+1}^{2} \hat{\psi}_{l+1}^{n}(E)+\varepsilon, \\
\hat{U}_{k}^{n}(E) & =\hat{u}^{n}(E)+\sum_{\substack{i=1 \\
i \neq k}}^{l} \hat{h}_{i}^{4} \hat{\psi}_{i}^{n}(E)+\frac{1}{16} \hat{h}_{k}^{4} \hat{\psi}_{k}^{n}(E)+\hat{h}_{l+1}^{2} \hat{\psi}_{l+1}^{n}(E)+\varepsilon, \\
k & =1, \ldots, l, \\
\hat{U}_{l+1}^{n}(E) & =\hat{u}^{n}(E)+\sum_{i=1}^{l} \hat{h}_{i}^{4} \hat{\psi}_{i}^{n}(E)+\frac{1}{4} \hat{h}_{l+1}^{2} \hat{\psi}_{l+1}^{n}(E)+\varepsilon .
\end{aligned}
$$

For any real numbers $\alpha_{i}, i=1,2,3$, multiply (5.2) by $\alpha_{1},(5.3)$ by $\alpha_{2}$ and (5.4) by $\alpha_{3}$, then add them together to get

$$
\begin{aligned}
& \alpha_{1} \hat{U}_{0}^{n}(E)+\alpha_{2} \sum_{k=1}^{l} \hat{U}_{k}^{n}(E)+\alpha_{3} \hat{U}_{l+1}^{n}(E) \\
& =\left(\alpha_{1}+\alpha_{2} l+\alpha_{3}\right) \hat{u}^{n}(E)+\left[\alpha_{1}+\alpha_{2}(l-1)+\alpha_{2} \frac{1}{16}+\alpha_{3}\right] \sum_{i=1}^{l} \hat{h}_{i}^{4} \hat{\psi}_{i}^{n}(E) \\
& \quad+\left(\alpha_{1}+\alpha_{2} l+\alpha_{3} \frac{1}{4}\right) \hat{h}_{l+1}^{2} \hat{\psi}_{l+1}^{n}(E)+\varepsilon
\end{aligned}
$$

Let

$$
\alpha_{1}+\alpha_{2} l+\alpha_{3}=1, \quad \alpha_{1}+\alpha_{2}(l-1)+\alpha_{2} \frac{1}{16}+\alpha_{3}=0, \quad \alpha_{1}+\alpha_{2} l+\alpha_{3} \frac{1}{4}=0 .
$$

Thus

$$
\alpha_{1}=-\frac{16}{15} l-\frac{1}{3}, \alpha_{2}=\frac{16}{15}, \alpha_{3}=\frac{4}{3} .
$$

Plugging (5.6) into (5.5), we finish the proof.

Based on Lemma 5.1, we get the splitting extrapolation formula for grid points in $\hat{\Omega}_{0}^{h}$ as follows:

$$
U_{0}(E)=\frac{16}{15} \sum_{i=1}^{l} \hat{U}_{i}^{n}(E)+\frac{4}{3} \hat{U}_{(l+1)}^{n}(E)+\left[-\frac{16}{15} l-\frac{1}{3}\right] \hat{U}_{0}^{n}(E) .
$$

(2) Type 1: grid points in $\bigcup_{i=1}^{l} \hat{\Omega}_{i}^{h} \backslash \hat{\Omega}_{0}^{h}$. Let $E_{1}$ and $E_{2}$ be two neighboring coarse grid points. Suppose $B$ is the midpoint of $E_{1} E_{2}$ and $B \in \hat{\Omega}_{i}^{h} \backslash \hat{\Omega}_{0}^{h}$.

Copyright (c) by SIAM. Unauthorized reproduction of this article is prohibited. 
LEMMA 5.2.

$$
\begin{gathered}
\hat{U}_{i}^{n}(B)-\frac{1}{30} \sum_{k=1}^{2}\left[\hat{U}_{0}^{n}\left(E_{k}\right)-\hat{U}_{i}^{n}\left(E_{k}\right)\right]-\frac{8}{15} \sum_{\substack{j=1 \\
j \neq i}}^{l} \sum_{k=1}^{2}\left[\hat{U}_{0}^{n}\left(E_{k}\right)-\hat{U}_{j}^{n}\left(E_{k}\right)\right] \\
\quad-\frac{2}{3} \sum_{k=1}^{2}\left[\hat{U}_{0}^{n}\left(E_{k}\right)-\hat{U}_{l+1}^{n}\left(E_{k}\right)\right] \\
(5.8)=\hat{u}^{n}(B)+\varepsilon+O\left(\hat{h}_{0}^{5}\right)+O\left(\hat{h}_{l+1}^{2} \hat{h}_{0}\right) .
\end{gathered}
$$

Proof. Because $E_{1}$ and $E_{2}$ are coarse grid points, (5.2), (5.3), and (5.4) are still true for them. Therefore, for all $j=1, \ldots, l, k=1,2$,

$$
\hat{U}_{0}^{n}\left(E_{k}\right)-\hat{U}_{j}^{n}\left(E_{k}\right)=\frac{15}{16} \hat{h}_{j}^{4} \hat{\psi}_{j}^{n}\left(E_{k}\right)+\varepsilon, \hat{U}_{0}^{n}\left(E_{k}\right)-\hat{U}_{l+1}^{n}\left(E_{k}\right)=\frac{3}{4} \hat{h}_{l+1}^{2} \hat{\psi}_{l+1}^{n}\left(E_{k}\right)+\varepsilon .
$$

Then

$\hat{h}_{j}^{4} \hat{\psi}_{j}^{n}\left(E_{k}\right)=\frac{16}{15}\left[\hat{U}_{0}^{n}\left(E_{k}\right)-\hat{U}_{j}^{n}\left(E_{k}\right)\right]+\varepsilon, \hat{h}_{l+1}^{2} \hat{\psi}_{l+1}^{n}\left(E_{k}\right)=\frac{4}{3}\left[\hat{U}_{0}^{n}\left(E_{k}\right)-\hat{U}_{l+1}^{n}\left(E_{k}\right)\right]+\varepsilon$.

Since

$$
\hat{\psi}_{j}^{n}(B)=\frac{\hat{\psi}_{j}^{n}\left(E_{1}\right)+\hat{\psi}_{j}^{n}\left(E_{2}\right)}{2}+O\left(\hat{h}_{0}\right), j=1, \ldots, l+1,
$$

we have

$$
\begin{gathered}
\hat{h}_{j}^{4} \hat{\psi}_{j}^{n}(B)=\frac{8}{15} \sum_{k=1}^{2}\left[\hat{U}_{0}^{n}\left(E_{k}\right)-\hat{U}_{j}^{n}\left(E_{k}\right)\right]+\varepsilon+O\left(\hat{h}_{0}^{5}\right), \\
\hat{h}_{l+1}^{2} \hat{\psi}_{l+1}^{n}(B)=\frac{2}{3} \sum_{k=1}^{2}\left[\hat{U}_{0}^{n}\left(E_{k}\right)-\hat{U}_{l+1}^{n}\left(E_{k}\right)\right]+\varepsilon+O\left(\hat{h}_{l+1}^{2} \hat{h}_{0}\right) .
\end{gathered}
$$

By Remark 4.1 and (4.23), we get

$$
\hat{U}_{i}^{n}(B)=\hat{u}^{n}(B)+\sum_{\substack{j=1 \\ j \neq i}}^{l} \hat{h}_{j}^{4} \hat{\psi}_{j}^{n}(B)+\frac{1}{16} \hat{h}_{i}^{4} \hat{\psi}_{i}^{n}(B)+\hat{h}_{l+1}^{2} \hat{\psi}_{l+1}^{n}(B)+\varepsilon
$$

Plugging (5.9) and (5.10) into (5.11), we finish the proof.

Based on Lemma 5.2, we get the splitting extrapolation formula for grid points in $\hat{\Omega}_{i}^{h} \backslash \hat{\Omega}_{0}^{h}$ as follows:

$$
\begin{aligned}
U_{1}(B)= & \hat{U}_{i}^{n}(B)-\frac{1}{30} \sum_{k=1}^{2}\left[\hat{U}_{0}^{n}\left(E_{k}\right)-\hat{U}_{i}^{n}\left(E_{k}\right)\right] \\
& -\frac{8}{15} \sum_{\substack{j=1 \\
j \neq i}}^{l} \sum_{k=1}^{2}\left[\hat{U}_{0}^{n}\left(E_{k}\right)-\hat{U}_{j}^{n}\left(E_{k}\right)\right]-\frac{2}{3} \sum_{k=1}^{2}\left[\hat{U}_{0}^{n}\left(E_{k}\right)-\hat{U}_{l+1}^{n}\left(E_{k}\right)\right] .
\end{aligned}
$$

(3) Type 2: Centers of rectangular elements. Suppose $C$ is the center of a rectangular element, $E_{k}(k=1, \ldots, 4)$ are the four vertices, and $B_{k}(k=1, \ldots, 4)$ are

Copyright ( by SIAM. Unauthorized reproduction of this article is prohibited. 
the midpoints of the four edges. First, $U_{0}\left(E_{k}\right)$ and $U_{1}\left(B_{k}\right)$ are computed according to (5.7) and (5.12). Then by using an incomplete biquadratic interpolation without term $x^{2} y^{2}$ [37], we get

$$
U_{2}(C)=\frac{1}{2} \sum_{k=1}^{4} U_{1}\left(B_{k}\right)-\frac{1}{4} \sum_{k=1}^{4} U_{0}\left(E_{k}\right) .
$$

(4) Type 3: Centers of rectangular parallelepiped elements. Suppose $D$ is the center of a rectangular parallelepiped element, $E_{k}(k=1, \ldots, 8)$ are the eight vertices, and $B_{k}(k=1, \ldots, 12)$ are the midpoints of the 12 edges. First, $U_{0}\left(E_{k}\right)$ and $U_{1}\left(B_{k}\right)$ are computed according to (5.7) and (5.12). Then by using an incomplete triquadratic interpolation without terms $x^{2} y^{2} z^{2}, x^{2} y^{2} z, x^{2} y z^{2}, x y^{2} z^{2}, x^{2} y^{2}, x^{2} z^{2}, y^{2} z^{2}$ [37], we get

$$
U_{3}(D)=\frac{1}{4} \sum_{k=1}^{12} U_{1}\left(B_{k}\right)-\frac{1}{4} \sum_{k=1}^{8} U_{0}\left(E_{k}\right) .
$$

6. A posteriori error estimate. In this section, we present some a posteriori error estimates. Suppose $E$ is a grid point in $\hat{\Omega}_{0}^{h}$. We first have an a posteriori error estimate for the approximation $\hat{U}_{i}^{n}(E)(i=0, \ldots, l+1)$.

Theorem 6.1.

$$
\begin{aligned}
\text { (6.1) }\left|\hat{U}_{0}^{n}(E)-\hat{u}^{n}(E)\right| \leq & \frac{16}{15} \sum_{i=1}^{l}\left|\hat{U}_{0}^{n}(E)-\hat{U}_{i}^{n}(E)\right|+\frac{4}{3}\left|\hat{U}_{0}^{n}(E)-\hat{U}_{l+1}^{n}(E)\right|+\varepsilon, \\
\left|\hat{U}_{i}^{n}(E)-\hat{u}^{n}(E)\right| \leq & \frac{16}{15} \sum_{\substack{j=1 \\
j \neq i}}^{l}\left|\hat{U}_{0}^{n}(E)-\hat{U}_{j}^{n}(E)\right|+\frac{4}{3}\left|\hat{U}_{0}^{n}(E)-\hat{U}_{l+1}^{n}(E)\right| \\
& +\frac{1}{15}\left|\hat{U}_{0}^{n}(E)-\hat{U}_{k}^{n}(E)\right|+\varepsilon, i=1, \cdots, l, \\
(6.2) \quad & \\
(6.3)\left|\hat{U}_{l+1}^{n}(E)-\hat{u}^{n}(E)\right| \leq & \frac{16}{15} \sum_{j=1}^{l}\left|\hat{U}_{0}^{n}(E)-\hat{U}_{j}^{n}(E)\right|+\frac{1}{3}\left|\hat{U}_{0}^{n}(E)-\hat{U}_{l+1}^{n}(E)\right|+\varepsilon .
\end{aligned}
$$

Proof. Using (5.2), (5.3), and (5.4) for all $j=1, \ldots, l$, we have

$$
\hat{U}_{0}^{n}(E)-\hat{U}_{j}^{n}(E)=\frac{15}{16} \hat{h}_{j}^{4} \hat{\psi}_{j}^{n}(E)+\varepsilon, \hat{U}_{0}^{n}(E)-\hat{U}_{l+1}^{n}(E)=\frac{3}{4} \hat{h}_{l+1}^{2} \hat{\psi}_{l+1}^{n}(E)+\varepsilon .
$$

Thus,

$$
\hat{h}_{j}^{4} \hat{\psi}_{j}^{n}(E)=\frac{16}{15}\left[\hat{U}_{0}^{n}(E)-\hat{U}_{j}^{n}(E)\right]+\varepsilon, \hat{h}_{l+1}^{2} \hat{\psi}_{l+1}^{n}(E)=\frac{4}{3}\left[\hat{U}_{0}^{n}(E)-\hat{U}_{l+1}^{n}(E)\right]+\varepsilon .
$$

Finally, plugging (6.4) back into (5.2), (5.3), and (5.4) and using the triangle inequality, we obtain the desired result.

By using the triangle inequality and (5.1), we get an a posteriori error estimate for the average of $\hat{U}_{j}^{n}(E)(j=1, \ldots, l+1)$ as follows.

TheOREM 6.2.

$$
\begin{aligned}
\left|\frac{1}{l+1} \sum_{i=1}^{l+1} \hat{U}_{i}^{n}(E)-\hat{u}^{n}(E)\right| \leq & \left|\frac{1}{l+1} \sum_{i=1}^{l+1} \hat{U}_{i}^{n}(E)-\hat{U}_{0}^{n}(E)\right|+\frac{16}{15} \sum_{i=1}^{l}\left|\hat{U}_{0}^{n}(E)-\hat{U}_{i}^{n}(E)\right| \\
& +\frac{4}{3}\left|\hat{U}_{0}^{n}(E)-\hat{U}_{l+1}^{n}(E)\right|+\varepsilon .
\end{aligned}
$$

Copyright (c) by SIAM. Unauthorized reproduction of this article is prohibited. 
7. Numerical experiments. In this section, we will present four numerical examples to illustrate the features of splitting extrapolation in this article. From the numerical results, especially the maximum error, we can see that splitting extrapolation improves the accuracy of the finite element approximations dramatically. We will see that our method is also efficient for solving discontinuous hyperbolic equations and nonlinear hyperbolic equations.

Example 1. Consider a hyperbolic equation with time-dependent coefficients and homogeneous Dirichlet boundary condition

$$
\left\{\begin{array}{lll}
\frac{\partial^{2} u}{\partial t^{2}}-\nabla\left(\left(t^{\frac{3}{2}}+x^{\frac{3}{2}}+y^{\frac{3}{2}}\right) \nabla u\right)=f(t, x, y) & \text { on } & {[0, T] \times \Omega} \\
u(0, x, y)=x(x-2) y(y-1), u_{t}(0, x, y)=x(x-2) y(y-1) & \text { on } & \bar{\Omega}, \\
u(t, x, y)=0 & \text { on } & {[0, T] \times \partial \Omega}
\end{array}\right.
$$

where $f(t, x, y)=x(x-2) y(y-1) e^{t}-2\left(y^{2}-y+x^{2}-x\right) t^{\frac{3}{2}} e^{t}-\left[(5 x-3) y(y-1)+2 x^{2}(x-\right.$ $2)] \sqrt{x} e^{t}-\left[2 y^{2}(y-1)+x(x-2)(5 y-1.5)\right] \sqrt{y} e^{t}, \Omega=(0,2) \times(0,1)$, and $T=1$. The exact solution is $u(t, x, y)=x(x-2) y(y-1) e^{t}$. First, we construct an initial domain decomposition $\bar{\Omega}=\bigcup_{s=1}^{2} \bar{\Omega}_{s}$, where $\Omega_{1}=(0,1) \times(0,1)$ and $\Omega_{2}=(1,2) \times(0,1)$. We design four independent step sizes as follows: $h_{i}(i=1,2)$ are the step sizes of $\Omega_{i}(i=1,2)$ in the $x$-direction; $h_{3}$ is the step size of both $\Omega_{1}$ and $\Omega_{2}$ in the $y$-direction; $h_{4}$ is the time step size.

In order to get the splitting extrapolation solution on the globally fine grid, we need only apply the biquadratic finite elements on the coarse grid and the locally fine grids. Therefore we do not compute the finite element solution at the nodes of the globally fine grid which are neither the nodes of the coarse grid nor the locally fine grids. In the following tables, let "**" denote these unknown results, "error of FE" denote the error of the finite element approximation, "error of SE" denote the error of the splitting extrapolation solution, and "max error" denote the maximum error on all nodes at all time steps. Let $h_{i}=\frac{1}{4}, i=1,2,3,4$. Then some error comparisons for Example 1 are provided in Table 1, which show that splitting extrapolation improves the accuracy dramatically.

TABLE 1

Numerical error comparison between FE and SE for Example 1.

\begin{tabular}{|l|l|l|l|}
\hline Grid points & Point type & Error of FE & Error of SE \\
\hline$(0.1250,0.5000, T)$ & type 0 & $-3.5592 \times 10^{-3}$ & $+4.3025 \times 10^{-6}$ \\
\hline$(1.0000,0.6250, T)$ & type 0 & $-1.2887 \times 10^{-2}$ & $+5.6532 \times 10^{-4}$ \\
\hline$(0.5625,0.3750, T)$ & type 1 & $-1.2191 \times 10^{-2}$ & $-7.6842 \times 10^{-6}$ \\
\hline$(1.0000,0.6875, T)$ & type 1 & $-1.1420 \times 10^{-2}$ & $+1.6120 \times 10^{-4}$ \\
\hline$(0.0625,0.9375, T)$ & type 2 & $* *$ & $-9.2033 \times 10^{-5}$ \\
\hline$(1.6875,0.4375, T)$ & type 2 & $* *$ & $-4.0577 \times 10^{-7}$ \\
\hline max error on coarse grid & & $-1.4938 \times 10^{-2}$ & $+1.3375 \times 10^{-3}$ \\
\hline max error on globally fine grid & & $* *$ & $+1.3375 \times 10^{-3}$ \\
\hline
\end{tabular}

In order to compare the errors we specify the analytic solution in our numerical examples. However, in the actual large scale computation we generally do not know the analytic solution. We need to use the a posteriori error estimates to control the errors of our numerical solutions. In Table 2 we show the maximum values of a posteriori error estimates at all coarse grid points, as discussed in section 6. Let APE1 be the maximum value of the a posteriori error estimate in (6.1), APE2, APE3, and APE4 be the maximum values of the a posteriori error estimate in (6.2) for $i=1,2,3$, 
respectively, APE5 be the maximum value of the a posteriori error estimate in (6.3), and APE6 be that of the a posteriori error estimate in (6.5).

TABLE 2

Some numerical results for a posteriori error estimates of Example 1.

\begin{tabular}{|l|l|l|l|l|l|}
\hline APE1 & APE2 & APE3 & APE4 & APE5 & APE6 \\
\hline $1.6031 \times 10^{-2}$ & $1.5938 \times 10^{-2}$ & $1.6027 \times 10^{-2}$ & $1.5952 \times 10^{-2}$ & $4.1980 \times 10^{-3}$ & $1.8983 \times 10^{-2}$ \\
\hline
\end{tabular}

In addition, Table 3 contains more numerical results with variable mesh parameters which verify the theoretical convergence analysis numerically. Let $E_{1}$ be the maximum error of the standard finite element solution at all the coarse grid points, $E_{2}$ be the maximum error of SE solution at all type 0 grid points (coarse grid points), $E_{3}$ be the maximum error of SE solution at all type 1 grid points, $E_{4}$ be the maximum error of SE solution at all type 2 grid points, $E_{5}$ be the error of the standard FE solution at point $\left(1, \frac{1}{2}\right)$, and $E_{6}$ be the error of SE solution at point $\left(1, \frac{1}{2}\right)$. Let $h_{b}$ be the basic grid parameter and choose $h_{i}=\frac{h_{b}}{4}, i=1,2,3$, and $h_{4}=h_{b}$. Using linear regression, we can see that the data in this table obey

$$
\begin{aligned}
& E_{1} \approx 0.2699 h^{2.1075}, E_{2} \approx 0.1129 h^{3.2687}, E_{3} \approx 0.1121 h^{3.2671}, \\
& E_{4} \approx 0.1132 h^{3.2725}, E_{5} \approx 0.2852 h^{2.1719}, E_{6} \approx 0.1408 h^{3.6326} .
\end{aligned}
$$

These linear regressions show that the splitting extrapolation solution is convergent at all types of points defined in section 5 with one higher order of convergence rate than the standard finite element solution. This numerically verifies our theoretical analysis in section 4 .

TABLE 3

Some numerical errors with variable grid parameters for Example 1.

\begin{tabular}{|l|l|l|l|l|l|l|}
\hline$h_{b}$ & $E_{1}$ & $E_{2}$ & $E_{3}$ & $E_{4}$ & $E_{5}$ & $E_{6}$ \\
\hline $1 / 4$ & $-1.5110 \times 10^{-2}$ & $1.2170 \times 10^{-3}$ & $1.2103 \times 10^{-3}$ & $1.2170 \times 10^{-3}$ & $-1.4801 \times 10^{-2}$ & $1.0170 \times 10^{-3}$ \\
\hline $1 / 8$ & $-3.2206 \times 10^{-3}$ & $1.3055 \times 10^{-4}$ & $1.3009 \times 10^{-4}$ & $1.2908 \times 10^{-4}$ & $-2.9379 \times 10^{-3}$ & $6.9894 \times 10^{-5}$ \\
\hline $1 / 12$ & $-1.3962 \times 10^{-3}$ & $3.2850 \times 10^{-5}$ & $3.2784 \times 10^{-5}$ & $3.2784 \times 10^{-5}$ & $-1.2435 \times 10^{-3}$ & $1.5421 \times 10^{-5}$ \\
\hline $1 / 16$ & $-7.8031 \times 10^{-4}$ & $1.2251 \times 10^{-5}$ & $1.2234 \times 10^{-5}$ & $1.2155 \times 10^{-5}$ & $-6.8222 \times 10^{-4}$ & $5.0867 \times 10^{-6}$ \\
\hline $1 / 20$ & $-4.9427 \times 10^{-4}$ & $6.2948 \times 10^{-6}$ & $6.2778 \times 10^{-6}$ & $6.2459 \times 10^{-6}$ & $-4.3268 \times 10^{-4}$ & $2.6124 \times 10^{-6}$ \\
\hline $1 / 24$ & $-3.4246 \times 10^{-4}$ & $3.6597 \times 10^{-6}$ & $3.6487 \times 10^{-6}$ & $3.6256 \times 10^{-6}$ & $-2.9924 \times 10^{-4}$ & $1.6823 \times 10^{-6}$ \\
\hline
\end{tabular}

As mentioned in Remark 2.1, in our theoretical analysis we consider only the hyperbolic equations with homogeneous Dirichlet boundary condition. However, the splitting extrapolation method can be used to handle the equations with nonhomogeneous Dirichlet boundary condition and mixed boundary condition. Here we present a numerical example for a hyperbolic equation with mixed boundary condition.

Example 2.

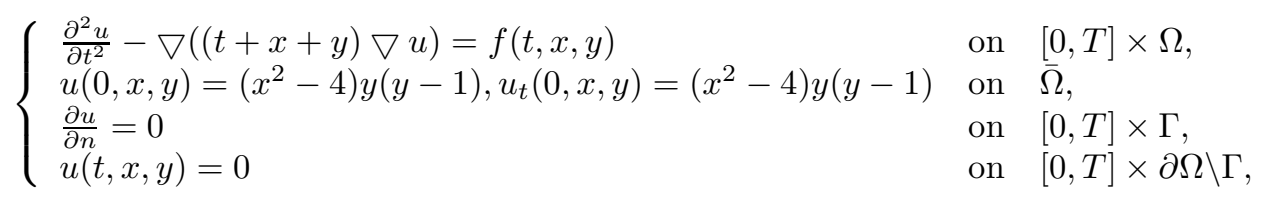

where $\Gamma$ is the left boundary of $\Omega$ and $\Omega$ is a curved quadrangle. Its bottom boundary is a line through $P_{1}=(0,0)$ and $P_{2}=(2,0)$, while its top boundary is a line through 
$P_{4}=(0,1)$ and $P_{3}=(2,1)$. The left-side boundary is a parabola through points $P_{1}$, $P_{8}=(-0.25,0.5)$, and $P_{4}$. The right-side boundary is a parabola through points $P_{2}$, $P_{6}=(2.25,0.5)$, and $P_{3}$. Let $P_{5}=(1,0), P_{7}=(1,1), P_{9}=\left(1, \frac{1}{2}\right)$, and $f(t, x, y)=$ $\left(x^{2}-4\right) y(y-1) e^{t}-2(2 x+y) y(y-1) e^{t}-(4 y+2 x-1)\left(x^{2}-4\right) e^{t}, T=1$. First, we construct an initial domain decomposition $\bar{\Omega}=\bigcup_{s=1}^{2} \bar{\Omega}_{s}$, where $\Omega_{1}=\Omega \bigcap\{x<1\}$ and $\Omega_{2}=\Omega \bigcap\{x>1\}$. With the $d$-quadratic isoparametric mapping, $\Omega, \Omega_{1}$, and $\Omega_{2}$ are mapped to $\hat{\Omega}=(0,2) \times(0,1), \hat{\Omega}_{1}=(0,1) \times(0,1)$, and $\hat{\Omega}_{2}=(1,2) \times(0,1)$ separately. Then we design four independent step sizes as follows: $h_{i}(i=1,2)$ are the step sizes of $\hat{\Omega}_{i}(i=1,2)$ in the $x$-direction; $h_{3}$ is the step size in the $y$-direction; $h_{4}$ is the time step size. Let $h_{i}=\frac{1}{4}, i=1,2,3,4$; then some error comparisons for Example 2 are shown in Table 4, which also show that splitting extrapolation improves the accuracy dramatically.

TABLE 4

Numerical error comparison between FE and SE for Example 2.

\begin{tabular}{|l|l|l|l|}
\hline Grid points & Point type & Error of FE & Error of SE \\
\hline$(0.0074,0.3750, T)$ & type 0 & $-3.1459 \times 10^{-2}$ & $-2.7882 \times 10^{-4}$ \\
\hline$(1.0000,0.7500, T)$ & type 0 & $-2.7605 \times 10^{-2}$ & $+4.1147 \times 10^{-5}$ \\
\hline$(0.3926,0.3125, T)$ & type 1 & $-4.7336 \times 10^{-2}$ & $+1.5628 \times 10^{-5}$ \\
\hline$(1.0000,0.6875, T)$ & type 1 & $-3.2676 \times 10^{-2}$ & $+1.4491 \times 10^{-4}$ \\
\hline$(0.2407,0.8125, T)$ & type 2 & $* *$ & $+3.7348 \times 10^{-6}$ \\
\hline$(1.5835,0.3125, T)$ & type 2 & $* *$ & $-4.3718 \times 10^{-4}$ \\
\hline max error on coarse grid & & $-5.8597 \times 10^{-2}$ & $+2.7915 \times 10^{-3}$ \\
\hline max error on globally fine grid & & $* *$ & $+2.7915 \times 10^{-3}$ \\
\hline
\end{tabular}

From the following example, we can see that splitting extrapolation is efficient for solving discontinuous problems if we regard the interfaces of the problem as the interfaces of the initial domain decomposition; see Remark 4.2.

Example 3. Consider an interface hyperbolic equation

$$
\left\{\begin{array}{lll}
\frac{\partial^{2} u}{\partial t^{2}}-\nabla(a(x, y) \nabla u)=f(t, x, y) & \text { on } & {[0, T] \times \Omega,} \\
u(0, x, y)=\Psi(x, y), u_{t}(0, x, y)=0 & \text { on } \bar{\Omega}, \\
u(t, x, y)=0 & \text { on } & {[0, T] \times \partial \Omega}
\end{array}\right.
$$

where

$$
a(x, y)=\left\{\begin{array}{lll}
r & \text { on } & \Omega_{1}, \\
1 & \text { on } & \Omega_{2},
\end{array}\right.
$$

and $\Omega$ is the same as that in Example 2. Let

$$
\begin{aligned}
& f(t, x, y)=\left\{\begin{array}{l}
-5 \cos t\left[3 x y(y-1)-1.5(r+1)(x-1)^{2} x y(y-1)\right] \\
+15 r(r+1) y(y-1)(3 x-2) \cos t-30 r\left[x-\frac{1}{2}(r+1) x(x-1)^{2}\right] \cos t, \\
x<1, \\
-5 \cos t\left[3 r x y(y-1)+3(1-r) y(y-1)-1.5(r+1)(x-1)^{2} x y(y-1)\right] \\
+15(r+1) y(y-1)(3 x-2) \cos t-30\left[r x+1-r-\frac{1}{2}(r+1) x(x-1)^{2}\right] \cos t \\
x \geq 1,
\end{array}\right. \\
& \Psi(x, y)= \begin{cases}5\left[3 x y(y-1)-1.5(r+1)(x-1)^{2} x y(y-1)\right], & x<1, \\
5\left[3 r x y(y-1)+3(1-r) y(y-1)-1.5(r+1)(x-1)^{2} x y(y-1)\right], & x \geq 1 .\end{cases}
\end{aligned}
$$


Here $r=0.5$. The domain decomposition and the design of independent step sizes are also the same as those in Example 2. The interface of the domain decomposition is exactly the same as the interface of $a(x, y)$, i.e., $x=1$. Let $h_{i}=\frac{1}{4}, i=1,2,3,4$. From the errors in Table 5, we can see that splitting extrapolation efficiently improves the accuracy for interface problems.

TABLE 5

Numerical error comparison between FE and SE for Example 3.

\begin{tabular}{|l|l|l|l|}
\hline Grid points & Point type & Error of FE & Error of SE \\
\hline$(1.4453,0.7500, T)$ & type 0 & $-7.1385 \times 10^{-2}$ & $-7.4270 \times 10^{-5}$ \\
\hline$(1.0000,0.1250, T)$ & type 0 & $-3.6289 \times 10^{-2}$ & $-3.8148 \times 10^{-4}$ \\
\hline$(1.2314,0.6250, T)$ & type 1 & $-9.7270 \times 10^{-2}$ & $-9.3504 \times 10^{-5}$ \\
\hline$(1.0000,0.4375, T)$ & type 1 & $-9.6935 \times 10^{-2}$ & $-2.1257 \times 10^{-3}$ \\
\hline$(2.1682,0.5625, T)$ & type 2 & $* *$ & $+2.9016 \times 10^{-5}$ \\
\hline$(0.0076,0.9375, T)$ & type 2 & $* *$ & $-1.9155 \times 10^{-4}$ \\
\hline max error on coarse grid & & $-1.0852 \times 10^{-1}$ & $-9.5752 \times 10^{-3}$ \\
\hline max error on globally fine grid & & $* *$ & $-9.5752 \times 10^{-3}$ \\
\hline
\end{tabular}

The following example demonstrates that our algorithm is also efficient for the nonlinear hyperbolic equations.

Example 4. Consider a nonlinear hyperbolic equation

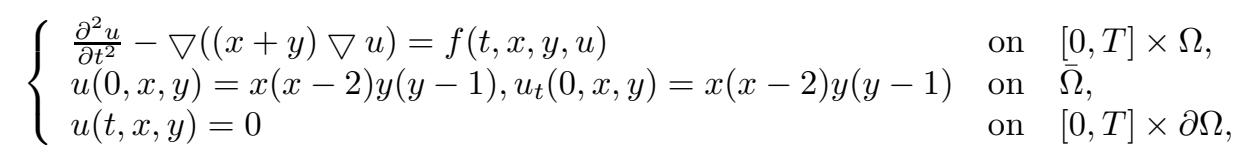

where $\Omega$ is the same as that in Example 1. Let $f(t, x, y, u)=u-\frac{(4 x+2 y-2)}{x^{2}(x-2)^{2} y(y-1) e^{t}} u^{2}-$ $(2 x+4 y-1) x(x-2) e^{t}$ and $T=1$. The domain decomposition and the design of independent step sizes are the same as those in Example 1. We use almost the same fully discrete scheme as $(2.9)$ except that we use $\hat{f}\left(t_{n}, \hat{U}^{n}\right)$ to replace $\hat{f}^{n}$ on the righthand side of the first equation. Let $h_{i}=\frac{1}{4}, i=1,2,3,4$; then similar numerical error comparisons are given in Table 6 to show the accuracy improvement.

TABLE 6

Numerical error comparison between $F E$ and $S E$ for Example 4.

\begin{tabular}{|l|l|l|l|}
\hline Grid points & Point type & Error of FE & Error of SE \\
\hline$(0.3750,0.7500, T)$ & type 0 & $+8.8577 \times 10^{-3}$ & $-1.1659 \times 10^{-5}$ \\
\hline$(1.0000,0.6250, T)$ & type 0 & $+2.3505 \times 10^{-2}$ & $+2.3972 \times 10^{-4}$ \\
\hline$(1.3125,0.5000, T)$ & type 1 & $+2.6245 \times 10^{-2}$ & $+9.4865 \times 10^{-6}$ \\
\hline$(1.0000,0.4375, T)$ & type 1 & $+2.6621 \times 10^{-2}$ & $+4.3551 \times 10^{-4}$ \\
\hline$(0.8125,0.9375, T)$ & type 2 & $* *$ & $+2.7611 \times 10^{-4}$ \\
\hline$(0.0625,0.3125, T)$ & type 2 & $* *$ & $+6.7384 \times 10^{-6}$ \\
\hline max error on coarse grid & & $+2.7194 \times 10^{-2}$ & $+9.3560 \times 10^{-4}$ \\
\hline max error on globally fine grid & & $* *$ & $+9.3560 \times 10^{-4}$ \\
\hline
\end{tabular}

8. Conclusions. To implement splitting extrapolation, the original problem was first converted into a new problem on polyhedrons by using domain decomposition and $d$-quadratic isoparametric mapping. Next, some independent grid parameters were chosen according to the dimension and interface of the problem, the shape and size of the domain, and the computers used. Then the multiparameter asymptotic 
expansion for the $d$-quadratic finite element solution error with respect to the independent grid parameters was established. Based on the expansion, we develop some splitting extrapolation formulas which can improve the order of accuracy. These formulas involve only linear combinations that are easy to implement. They generate an approximation with higher accuracy on a globally fine grid while requiring only some approximations from a set of smaller discrete subproblems on different coarser grids. Additionally, the multiparameter expansion requires only the local smoothness of the solutions, i.e., the smoothness of the solutions in each subdomain. Therefore, splitting extrapolation is efficient for solving discontinuous problems if we regard the interfaces of the problems as the interfaces of the initial domain decomposition. The numerical results also showed that the algorithm is efficient for solving nonlinear hyperbolic equations. The corresponding proof for the nonlinear case leads to interesting future research projects.

Finally, using ideas similar to those presented in $[18,27,38,54]$, we can compute the solutions $\hat{U}_{i}^{n}, i=0, \ldots, l+1$, to the smaller discrete subproblems in parallel. This method also possesses a high degree of parallelism because those subproblems are independent of each other and their loads are balanced if we construct the domain decomposition proportionally and choose independent variables properly.

\section{REFERENCES}

[1] G. A. BAKER, Error estimates for finite element methods for second order hyperbolic equations, SIAM J. Numer. Anal., 13 (1976), pp. 564-576.

[2] G. A. Baker And V. A. Dougalis, The effect of quadrature errors on finite element approximations for second order hyperbolic equations, SIAM J. Numer. Anal., 13 (1976), pp. $577-598$.

[3] G. A. Baker And V. A. Dougalis, On the $L_{\infty}$-convergence of Galerkin approximations for second-order hyperbolic equations, Math. Comp., 34 (1980), pp. 401-424.

[4] F. G. Basombrío, L. Guarracino, and M. J. Vénere, A non-iterative algorithm based on Richardson's extrapolation. Application to groundwater flow modelling, Internat. J. Numer. Methods Engrg., 65 (2006), pp. 1088-1112.

[5] P. J. Bickel And J. A. Yahav, Richardson extrapolation and the bootstrap, J. Amer. Statist. Assoc., 83 (1988), pp. 387-393.

[6] H. Blum, Q. Lin, And R. Rannacher, Asymptotic error expansion and Richardson extrapolation for linear finite elements, Numer. Math., 49 (1986), pp. 11-37.

[7] H. Bungartz, M. GRIebel, And U. RüDE, Extrapolation, combination, and sparse grid techniques for elliptic boundary value problems, Comput. Methods Appl. Mech. Engrg., 116 (1994), pp. 243-252.

[8] Y. CAO, X.-M. HE, AND T. Lü, A splitting extrapolation for solving nonlinear elliptic equations with d-quadratic finite elements, J. Comput. Phys., 228 (2009), pp. 109-122.

[9] C. M. Chen, F. Liu, And V. Anh, A Fourier method and an extrapolation technique for Stokes' first problem for a heated generalized second grade fluid with fractional derivative, J. Comput. Appl. Math., 223 (2009), pp. 777-789.

[10] P. G. Ciarlet, The Finite Element Method for Elliptic Problems, North-Holland, Amsterdam, 1978.

[11] J. Douglas, T. Dupont, And M. F. Wheeler, A quasi-projection analysis of Galerkin methods for parabolic and hyperbolic equations, Math. Comp., 32 (1978), pp. 345-362.

[12] T. Dupont, $L^{2}$-estimates for Galerkin methods for second order hyperbolic equations, SIAM J. Numer. Anal., 10 (1973), pp. 880-889.

[13] J. DutKa, Richardson extrapolation and Romberg integration, Historia Math., 11 (1984), pp. 321.

[14] G. Fairweather, Q. Lin, Y. Lin, J. Wang, And S. Zhang, Asymptotic expansions and Richardson extrapolation of approximate solutions for second order elliptic problems on rectangular domains by mixed finite element methods, SIAM J. Numer. Anal., 44 (2006), pp. $1122-1149$.

[15] R. Fössmeier, On Richardson extrapolation for finite difference methods on regular grids, Numer. Math., 55 (1989), pp. 451-462. 
[16] T. GevecI, On the convergence of Galerkin approximation schemes for second-order hyperbolic equations in energy and negative norms, Math. Comp., 42 (1984), pp. 393-415.

[17] G. HAN AND R. WANG, Richardson extrapolation of iterated discrete Galerkin solution for twodimensional Fredholm integral equations, J. Comput. Appl. Math., 139 (2002), pp. 49-63.

[18] X.-M. He AND T. Lü, Splitting extrapolation method for solving second order parabolic equations with curved boundaries by using domain decomposition and d-quadratic isoparametric finite elements, Int. J. Comput. Math., 84 (2007), pp. 767-781.

[19] J. HuAng AND T. Lü, Splitting extrapolations for solving boundary integral equations of linear elasticity Dirichlet problems on polygons by mechanical quadrature methods, J. Comput. Math., 24 (2006), pp. 9-18.

[20] S. Jia, H. XIE, X. YIN, AND S. GaO, Approximation and eigenvalue extrapolation of biharmonic eigenvalue problem by nonconforming finite element methods, Numer. Methods Partial Differential Equations, 24 (2008), pp. 435-448.

[21] B. Johansson And V. Christer, The use of Richardson extrapolation for the numerical solution of low Mach number flow in confined regions, J. Sci. Comput., 8 (1993), pp. 307-340.

[22] C. Johnson, Numerical Solution of Partial Differential Equations by the Finite Element Method, Cambridge University Press, Cambridge, UK, 1987.

[23] D. C. JoYCE, Survey of extrapolation processes in numerical analysis, SIAM Rev., 13 (1971), pp. $435-490$.

[24] M. JUNG AND U. RÜDE, Implicit extrapolation methods for multilevel finite element computations, SIAM J. Sci. Comput., 17 (1996), pp. 156-179.

[25] M. JUng AND U. RÜDE, Implicit extrapolation methods for variable coefficient problems, SIAM J. Sci. Comput., 19 (1998), pp. 1109-1124.

[26] S. Larsson and V. Thomée, Partial Differential Equations with Numerical Methods, Texts Appl. Math. 45, Springer-Verlag, Berlin, 2003.

[27] X. LiaO And A. Zhou, A multi-parameter splitting extrapolation and a parallel algorithm for elliptic eigenvalue problem, J. Comput. Math., 16 (1998), pp. 213-220.

[28] C. B. Liem, T. Lü, And T. M. Shin, The Splitting Extrapolation Method. A New Technique in Numerical Solution of Multidimensional Problems, Ser. Appl. Math. 7, World Scientific, River Edge, NJ, 1995.

[29] Q. Lin AND J. Lin, Extrapolation of the bilinear element approximation for the Poisson equation on anisotropic meshes, Numer. Methods Partial Differential Equations, 23 (2007), pp. 960967.

[30] Q. Lin AND T. Lü, The splitting extrapolation method for multidimensional problem, J. Comput. Math., 1 (1983), pp. 45-51.

[31] Q. Lin, T. LÜ, AND S. SHEn, Maximum norm estimate extrapolation and optima points of stresses for the finite element methods on the strongly triangulation, J. Comput. Math., 1 (1983), pp. 376-383.

[32] Q. Lin, I. H. SlOAN, AND R. XIE, Extrapolation of the iterated-collocation method for integral equations of the second kind, SIAM J. Numer. Anal., 27 (1990), pp. 1535-1541.

[33] Q. J. Lin AND Q. D. ZHU, Unidirectional extrapolations of finite difference and finite elements, Engrg. Math., 1 (1984), pp. 1-12 (in Chinese).

[34] T. Lin, Y. Lin, M. RaO, AND S. Zhang, Petrov-Galerkin methods for linear Volterra integrodifferential equations, SIAM J. Numer. Anal., 38 (2000), pp. 937-963.

[35] Y. LiU AND T. Lü, Mechanical quadrature methods and their extrapolation for solving first kind Abel integral equations, J. Comput. Appl. Math., 201 (2007), pp. 300-313.

[36] T. Lü, Correction and splitting extrapolation methods for the collocation solutions of two point boundary value problems, Adv. in Math. (Beijing), 16 (1987), pp. 391-396 (in Chinese).

[37] T. Lü AND Y. Feng, Splitting extrapolation based on domain decomposition for finite element approximations, Sci. China Ser. E, 40 (1997), pp. 144-155.

[38] T. LÜ AND J. LU, Splitting extrapolation for solving second order elliptic systems with curved boundary in $\Re^{d}$ by using d-quadratic isoparametric finite element, Appl. Numer. Math., 40 (2002), pp. 467-481.

[39] T. Lü, T. M. Shin, ANd C. B. LiEm, An analysis of the splitting extrapolation for multidimensional problem, Systems Sci. Math. Sci., 3 (1990), pp. 261-272.

[40] T. Lü, T. M. Shin, And C. B. Liem, Splitting Extrapolation and Combination Techniques, Scientific Press, Beijing, 1998 (in Chinese).

[41] G. Marchuk and V. Shaİdurov, Difference Methods and Their Extrapolations, Appl. Math. (N. Y.) 19, Springer-Verlag, New York, 1983.

[42] M. D. MARcozzI, Extrapolation discontinuous Galerkin method for ultraparabolic equations, J. Comput. Appl. Math., 224 (2009), pp. 679-687.

[43] P. NeittaAnmäki And Q. Lin, Acceleration of the convergence in finite difference methods

Copyright (c) by SIAM. Unauthorized reproduction of this article is prohibited. 
by predictor corrector and splitting extrapolation methods, J. Comput. Math., 5 (1987), pp. $181-190$.

[44] K. Rahul and S. N. Bhattacharyya, One-sided finite-difference approximations suitable for use with Richardson extrapolation, J. Comput. Phys., 219 (2006), pp. 13-20.

[45] S. A. Richards, Completed Richardson extrapolation in space and time, Comm. Numer. Methods Engrg., 13 (1997), pp. 573-582.

[46] U. RüDE, Book review: The Splitting Extrapolation Method (C. B. Liem, T. Lü, and T. M. Shih), SIAM Rev., 39 (1997), pp. 161-162.

[47] U. RÜDE AND A. Zhou, Multi-parameter extrapolation methods for boundary integral equations. Numerical treatment of boundary integral equations, Adv. Comput. Math., 9 (1998), pp. $173-190$.

[48] A. SIDI, A complete convergence and stability theory for a generalized Richardson extrapolation process, SIAM J. Numer. Anal., 34 (1997), pp. 1761-1778.

[49] A. SIDI, The Richardson extrapolation process with a harmonic sequence of collocation points, SIAM J. Numer. Anal., 37 (2000), pp. 1729-1746.

[50] J. WANG, Superconvergence and extrapolation for mixed finite element methods on rectangular domains, Math. Comp., 56 (1991), pp. 477-503.

[51] Y. WAng AND J. Zhang, Sixth order compact scheme combined with multigrid method and extrapolation technique for 2 D Poisson equation, J. Comput. Phys., 228 (2009), pp. 137146.

[52] M. F. Wheeler, $L_{\infty}$ estimates of optimal orders for Galerkin methods for one-dimensional second order parabolic and hyperbolic equations, SIAM J. Numer. Anal., 10 (1973), pp. 908913.

[53] X. Yin, H. XiE, S. JiA, AND S. GaO, Asymptotic expansions and extrapolations of eigenvalues for the Stokes problem by mixed finite element methods, J. Comput. Appl. Math., 215 (2008), pp. 127-141.

[54] A. Zhou, C. B. Liem, T. M. Shin, And T. Lü, A multi-parameter splitting extrapolation and a parallel algorithm, Systems Sci. Math. Sci., 10 (1997), pp. 253-260.

Copyright (c) by SIAM. Unauthorized reproduction of this article is prohibited. 